\title{
Weighted association schemes, fusions, and minimal coherent closures
}

\author{
A. D. Sankey
}

Received: 17 July 2013 / Accepted: 16 August 2014 / Published online: 9 September 2014

(C) Springer Science+Business Media New York 2014

\begin{abstract}
A weighted association scheme is a scheme with an edge weight function, which for our purposes will take values \pm 1 . When the scheme has a coherent fusiona merging of classes resulting in another association scheme-the edge weights on the fusion scheme are inherited. The reverse process involves the coherent closure of a weighted scheme: the smallest coherent algebra containing the weighted adjacency matrices. The weight function applied to this closure is necessarily trivial, meaning constant on classes of the associated configuration. In this work there are two main objects of study: minimal rank coherent closures of strongly regular graphs with regular weights; and strongly regular graphs with regular weights which are obtained as fusions of association schemes with trivial regular weights. Both of these extend work of D. Taylor on regular two-graphs and their interactions with strongly regular graphs. We obtain regular weights on strongly regular graphs with 125 and 256 vertices as fusions of rank 5 weighted schemes, and a family of rank 6, primitive, non-metric schemes which are minimal-rank closures of weighted lattice graphs. Our main result is the classification of these coherent closures of rank 4. They are imprimitive rank 4 association schemes, which have been studied generally by E. R. van Dam, and Y. Chang and T. Huang.
\end{abstract}

Keywords Association scheme - Coherent configuration - Coherent closure · Weisfeiler-Lehman stabilization · Regular weight

Mathematics Subject Classification $\quad 05 \mathrm{C} 12 \cdot 05 \mathrm{C} 22 \cdot 05 \mathrm{C} 50 \cdot 05 \mathrm{E} 30$

\footnotetext{
A. D. Sankey $(\varangle)$

Department of Mathematics and Statistics, University of New Brunswick, P.O. Box 4400, Fredericton, NB E3B 5A3, Canada e-mail: asankey@unb.ca
} 


\section{Introduction}

In a 2007 paper on association schemes of order 28, Klin et al. [17] offer a unifying perspective on schemes of a given order. Defining a coherent configuration (CC) of rank 112 on 28 points called $\operatorname{AP}(2)$, the authors describe a variety of mergings, or fusions, of classes which give association schemes. In this way, a number of schemes on 28 points of various ranks are brought together under one theoretical umbrella, with a common parent configuration.

The goal of the present work is twofold. First, in the spirit of Klin et al., we aim to describe some schemes as fusions of higher rank CCs. While the approach to AP(2) makes use of its automorphism group, our primary tool will be regular weights on SRGs as defined by Higman in [14]. Constructing such a weight with values \pm 1 on a "child" scheme (or configuration) gives an associative algebra, spanned by the weighted adjacency matrices, which is not in general closed under the entry-wise product thus is not in itself a coherent algebra. Using the stabilization, or coherent closure algorithm, we may however determine the smallest coherent algebra containing it, and we find that this algebra contains the Bose-Mesner algebra (or coherent algebra) of the child scheme. Hence we have found a refinement or parent configuration via the weight. This approach provides a method for identifying, and possibly building higher rank CCs as fissions of known weighted ones.

This aim also extends a result of D. Taylor on regular two-graphs. A regular twograph is equivalent to a regular weight on a rank 2 CC (i.e., a complete graph), and in fact it was with an eye to generalizing Taylor's regular two-graphs that Higman defined regular weights on CCs. Taylor considered (see Sect. 6) the regular two-graphs with strongly regular graphs (SRGs) in their switching classes. Put another way, these regular weights on the complete graph have coherent closures of rank 3, giving SRGs. To extend his result, we ask: When does a regular weight of whatever rank, on a child configuration, have minimal closure? We will see some examples in the form of SRGs with regular weights having coherent closures of ranks 4, 5, 6 .

Our second aim is to extend a related result of Taylor's. Here, he asked which SRGs determine a regular two-graph. Taking the Seidel adjacency matrix of the SRG, we look to see whether the switching class of that graph is a regular two-graph. In our terminology, the Seidel matrix represents a trivial weight on the SRG: one in which all edges are weighted -1 and all non-edges +1 . When the SRG and its complement are fused to the complete graph, the weight is correspondingly fused, to a weight on $K_{n}$. This fusion is coherent when the result is a regular weight, giving a regular two-graph. We extend Taylor's result by asking when does a trivial weight on a parent CC have a coherent fusion, to a regular weight on a CC. Thus we have a procedure for building non-trivial regular weights from trivial ones on known schemes. For example, a new regular weight on $\operatorname{SRG}(126,25,8,4)$ is obtained from a trivial weight on the Odd graph on 9 points, a distance regular graph of diameter 4 , by merging the distance 1 and 2 graphs of the scheme.

Section 2 contains preliminaries on CCs, regular weights, two-graphs and coherent closures. In Sects. 3 and 4 we introduce coherent closure and fusion in the context of regular weights. In Sect. 5 we present some detailed examples to motivate the discussion that follows and clarify the definitions. In Sect. 6, we treat the rank 2 case, 
beginning with Taylor's results on SRGs and regular two-graphs. A family of rank 6 closures is presented in Sect. 7. In Sect. 8 we classify the rank 4 coherent closures.

GAP [29], sage [27], and Maple ${ }^{\mathrm{TM}}$ [18] were used to perform computations throughout. Brouwer's parameter tables for SRGs [4] and distance-regular graphs [3] were accessed, as were the association schemes of small order provided by Hanaki and Miyamoto [10].

\section{Preliminaries}

\subsection{Coherent configurations}

The concept of a coherent configuration was developed independently by Weisfeiler and Lehman in [32] and by Higman in [11,13]. A good introduction to the topic may be found in [16]. We consider an algebra $\mathcal{A}$ of $n$ by $n$ matrices, over $\mathbb{C}$ with the following properties:

(i) $\mathcal{A}$ has a distinguished basis of $(0,1)$ matrices $\left\{\mathbf{A}_{0}, \mathbf{A}_{1}, \ldots, \mathbf{A}_{r-1}\right\}$;

(ii) For each $i$ there exists $i^{*} \in\{0, \ldots, r-1\}$ such that $\mathbf{A}_{i}^{T}=\mathbf{A}_{i^{*}}$;

(iii) $\sum_{i=0}^{r-1} \mathbf{A}_{i}=\mathbf{J}$ where $\mathbf{J}$ is the all-ones matrix;

(iv) $\mathbf{I} \in \mathcal{A}$.

This defines the coherent algebra $\mathcal{A}$ of degree $n$ and rank $r$, along with its standard basis. We define the support of a matrix to be the set of indices corresponding to nonzero entries. Thus (iii) indicates that the $\mathbf{A}_{i}$ have non-overlapping supports. Note that properties (i), (iii), and (iv) imply the existence of a subset $S$ of the index set $\mathcal{I}$, such that $\mathbf{I}=\sum_{i \in S} \mathbf{A}_{i}$.

Each of the basis matrices may be thought of as the adjacency matrix of a graph or, equivalently, a relation, with rows and columns labelled by a common set of vertices $X$. The graph with adjacency matrix $\mathbf{A}_{i}$ will be denoted $\Gamma_{i}$, and the corresponding binary relation $f_{i}$. For any binary relation $f$ on $X$, we define $f^{T}:=\left\{(y, x) \in X^{2} \mid(x, y) \in f\right\}$ and $f(x):=\{y \in X \mid(x, y) \in f\}$.

The fact that $\mathcal{A}$ is a matrix algebra means that there exist complex numbers $p_{i j}^{k}$ (the structure constants, or intersection numbers) such that

$$
\mathbf{A}_{i} \mathbf{A}_{j}=\sum_{k} p_{i j}^{k} \mathbf{A}_{k}
$$

In fact, the intersection numbers are all integers with a combinatorial interpretation. The constant $p_{i j}^{k}$ counts the number of $i-j$ paths from a vertex $x$ to a vertex $z$, given that $(x, z) \in f_{k}$ and this number is necessarily independent of the choice of edge in $\Gamma_{k}$. It is convenient to denote each instance of an $i-j$ path by a triangle $(x, y, z)$ of type $(i, j, k)$. That is, $(x, y, z) \in X^{3}$ is a triangle of type $(i, j, k)$ if $(x, y) \in f_{i},(y, z) \in f_{j}$, and $(x, z) \in f_{k}$. 
Definition 1 A coherent configuration or $\mathrm{CC}\left(X,\left\{f_{i}\right\}_{i \in \mathcal{I}}\right)$ is a vertex set $X$ together with a set of relations $\left\{f_{i}\right\}$ on $X$, indexed by $\mathcal{I}$, whose adjacency matrices form the standard basis of a coherent algebra.

The definitions may be formulated the other way around as in [13], with combinatorial axioms replacing (i-iv) and implying the existence of a coherent algebra.

Commutativity of $\mathcal{A}$ is equivalent to the condition that $p_{i j}^{k}=p_{j i}^{k}$ for all $i, j, k \in$ $\{0,1, \ldots, r-1\}$. The algebra is symmetric if the elements of the basis are, and this equates to $i^{*}=i$ for all $i \in\{0,1, \ldots, r-1\}$.

If $|S|=1$ then one of the basis matrices is the identity matrix and we denote it by $\mathbf{A}_{0}$. In this case, the configuration is said to be homogenous. Acknowledging that definitions in the literature vary on this point, we will call a homogeneous, symmetric (hence commutative) $\mathrm{CC}$ an association scheme. The coherent algebra corresponding to an association scheme is usually called the Bose-Mesner algebra. Some excellent references for schemes are $[2,5,6,9,19]$. A scheme is imprimitive if any of its nonidentity relations is not connected.

Clearly by (i) and (iii), a coherent algebra is closed under entry-wise (or Hadamard, or Schur) multiplication. In fact, $\mathbf{A}_{i} \circ \mathbf{A}_{j}=0$ for $i \neq j$ and $\mathbf{A}_{i} \circ^{2}=\mathbf{A}_{i}$. Thus the standard basis consists of idempotents with respect to this multiplication. In the commutative case, there is a dual basis of matrices which are idempotents with respect to ordinary matrix multiplication.

For the schemes and weighted schemes discussed here, it is possible to determine the character-multiplicity table. We define the intersection matrices $\mathbf{M}_{j}$, by $\mathbf{M}_{j}:=\left(p_{i j}^{k}\right)$ thus the map

$$
\gamma: \mathbf{A}_{j} \mapsto \mathbf{M}_{j}
$$

is the right regular representation of $\mathcal{A}$. As its image is isomorphic to $\mathcal{A}$ as a matrix algebra over $\mathbb{C}$, we obtain the eigenvalues of $\mathbf{A}_{j}$ from $\mathbf{M}_{j}$. Thus a rank $r$ scheme has standard basis elements with at most $r$ distinct eigenvalues. Using the fact that the $\mathbf{A}_{i}$ are simultaneously diagonalizable when the $\mathrm{CC}$ is commutative, we may compute the common multiplicities of the eigenvalues for the scheme. More detail may be found in $[2,5,9,13,14]$.

In the association scheme literature, a rank $r$ scheme is often referred to as an $(r-1)$-class scheme: 'rank' counts the trivial relation, while the number of 'classes' does not.

Commutative schemes which have the metric or P-polynomial property are synonymous with distance-regular graphs (DRGs) which are well documented in [5,9], for example, and in the new survey [31]. The DRGs of diameter 2 are the SRGs. Some familiarity with these structures is assumed.

\subsection{Fusion and closure}

A fusion or merging of relations (classes) in a $\mathrm{CC}$ corresponds to forming unions of (edge sets in) the associated graphs. A fusion will be deemed coherent if the resulting 
configuration is coherent. A coherent fission or refinement is the opposite: the edge set of each basis graph is partitioned in such a way that the corresponding relations form a CC.

The rank $2 \mathrm{CC}$ represented by $K_{n}$ is the minimum element in the lattice of all CCs on a given vertex set $X$ of size $n$ [13, Prop.3]. The maximum element has rank $n^{2}$, with the full matrix algebra $M_{X}(\mathbb{C})$ as its coherent algebra.

Definition 2 The coherent closure (CCL) or Weisfeiler-Lehman stabilization of a set of matrices is the intersection of all coherent algebras containing it.

We note that any graph $\Gamma$, via the CCL of its adjacency matrix, generates a coherent algebra, the underlying $\mathrm{CC}$ of which contains a set of relations whose union is the edge set of $\Gamma$.

We refer to the CCL of a set of graphs or relations on $X$ in the natural way, as the CC underlying the CCL of its set of adjacency matrices. There is a well-known algorithm for computing the CCL of a finite set of matrices (see $[1,32]$ for more). The CCL is a refinement of the original scheme which may, of course, be recovered through fusion.

\subsection{Two-graphs}

The set of all graphs that are equivalent to a given graph $\Gamma$ under Seidel switching, the switching class of $\Gamma$, is called a two-graph. See [24-26] for excellent surveys of two-graphs. Combinatorially, we define a two-graph as a distinguished set of 3-sets (the coherent triples) from a finite set (here, the vertex set of a graph), with the property that any 4-set contains an even number of coherent triples.

The regular two-graphs [28] have the additional property that every pair is contained in the same number of coherent triples. It is easy to see that switching preserves this constant. The Seidel matrix of any graph in the class of a regular two-graph has a quadratic minimal polynomial.

In the discussion of weights that follows, it will be convenient to consider a twograph in which the coherent triples are determined by a 3 -cochain, denoted $\delta \omega$, taking values in $\{ \pm 1\}$. We may define the coherent triples to be

$$
\left\{(x, y, z) \in X^{3} \mid \delta \omega(x, y, z)=-1\right\}
$$

hence the two-graph is regular if every pair $(x, z)$ lies in the same number of triangles $(x, y, z)$ with $\delta \omega(x, y, z)=-1$.

\subsection{Weights}

For a simple example, consider the pentagon and its complement, with edge weights given by the matrices below. 


$$
\mathbf{A}_{1}^{\omega}=\left(\begin{array}{rrrrr}
0 & 1 & & & 1 \\
1 & 0 & -1 & & \\
& -1 & 0 & 1 & \\
& & 1 & 0 & -1 \\
1 & & & -1 & 0
\end{array}\right) \quad \mathbf{A}_{2}^{\omega}=\left(\begin{array}{rrrrr}
0 & & -1 & -1 & \\
& 0 & & -1 & 1 \\
-1 & & 0 & & -1 \\
-1 & -1 & & 0 & \\
& 1 & -1 & & 0
\end{array}\right)
$$

Regularity of the weight is defined precisely below. It implies that the span of these matrices, along with the identity matrix representing the trivial relation, is closed under multiplication. This weight is regular, as can easily be checked by $\mathbf{A}_{1}^{\omega 2}$ $=2 \mathbf{I}+\mathbf{A}_{2}^{\omega}, \mathbf{A}_{1}^{\omega} \mathbf{A}_{2}^{\omega}=\mathbf{A}_{1}^{\omega}+\mathbf{A}_{2}^{\omega}$, and $\mathbf{A}_{2}^{\omega 2}=2 \mathbf{I}+\mathbf{A}_{1}^{\omega}$. In fact, this weight is equivalent to the trivial all-ones weight under switching on vertices 3 and 4 . In contrast, the matrices below define a weight that is not regular on the pentagon, as $\left(\mathbf{A}_{1}^{\omega}\right)^{2}$ does not lie in the span of $\mathbf{I}, \mathbf{A}_{1}^{\omega}$ and $\mathbf{A}_{2}^{\omega}$.

$$
\mathbf{A}_{1}^{\omega}=\left(\begin{array}{rrrrr}
0 & -1 & & & -1 \\
-1 & 0 & 1 & & \\
& 1 & 0 & 1 & \\
& & 1 & 0 & -1 \\
-1 & & & -1 & 0
\end{array}\right) \quad \mathbf{A}_{2}^{\omega}=\left(\begin{array}{rrrrr}
0 & & -1 & -1 & \\
& 0 & & 1 & 1 \\
-1 & & 0 & & 1 \\
-1 & 1 & & 0 & \\
& 1 & 1 & & 0
\end{array}\right)
$$

Regular weights on CCs are defined in [14] and earlier in [12]. In this work, we specify to weights with values \pm 1 and thus will define the terminology accordingly. In particular, all of our weights will have full support.

Let $X$ be a nonempty, finite set and let $U:=\{ \pm 1\}$. We say $f: X^{p} \rightarrow U$ is a $p$-cochain if, for $x=\left(x_{1}, x_{2}, \ldots, x_{p}\right) \in X^{p}$

1. $f(x)=1$ when $x_{i}=x_{j}$ for some $i \neq j$;

2. $f(y)=f(x)$ when $y$ results from $x$ by interchanging $x_{i}$ and $x_{j}$ for some $i \neq j$.

The coboundary of $f$ is

$$
\delta f(x)=\prod_{i=0}^{p} f\left(x_{0}, \ldots, x_{i-1}, x_{i+1}, \ldots, x_{p}\right) \text { for } x \in X^{p+1}
$$

and the coboundary operator $\delta: C^{p}(U) \rightarrow C^{p+1}(U)$ is a homomorphism from the group of $p$-cochains to the group of $(p+1)$-cochains on $U$, where the group operation is

$$
(f \cdot g)(x)=f(x) g(x) .
$$

In the standard way, we define the $p$-coboundaries:

$$
B^{p}(U):=\left\{\delta f \mid f \in C^{p-1}(U)\right\}
$$

and the $p$-cocycles:

$$
Z^{p}(U):=\left\{f \in C^{p}(U) \mid \delta f=1\right\}
$$


and observe that $\delta^{2} f=1$ and $Z^{p}(U)=B^{p}(U)$ for $p \geq 2$ [14, p. 503].

A weight on $X$ with values in $U$ is defined as a 2-cochain $\omega \in C^{2}(X, U)$, and may be thought of as a matrix with rows and columns indexed by $X$. When $X$ is the vertex set of a CC $\mathcal{X}$, we say $\omega$ is defined on $\mathcal{X}$. We set

$$
\mathbf{A}_{i}^{\omega}:=\omega \circ \mathbf{A}_{i}
$$

for each $i$. These weighted adjacency matrices represent weighted graphs, where $\omega(x, y)$ is the weight on the edge $(x, y)$, which of course belongs to exactly one of the relations.

We are interested in coherent weights, those for which the linear span of the weighted adjacency matrices is closed under matrix multiplication. This requirement implies the existence of structure constants $\beta_{i j}^{k}$ for the weighted coherent algebra, giving the weighted analogue of Eq. (1):

$$
\mathbf{A}_{i}^{\omega} \mathbf{A}_{j}^{\omega}=\sum_{k} \beta_{i j}^{k} \mathbf{A}_{k}^{\omega}
$$

Because $\omega$ takes values \pm 1 , these structure constants are integers.

Definition 3 A weight $\omega$ with values in $U=\{ \pm 1\}$ is coherent on a $\mathrm{CC} \mathcal{X}$ if there exist integers $\beta_{i j}^{k}$ such that the weighted adjacency matrices satisfy (2).

Given a coherent weight, we use the notation $\mathcal{A}^{\omega}$ for the weighted coherent algebra, that is the matrix algebra over $\mathbb{C}$ spanned by $\left\{\mathbf{A}_{i}^{\omega}\right\}$. Note that $\mathcal{A}^{\omega}$ is not, in general, a coherent algebra as it is not closed under entry-wise multiplication.

If the underlying CC has rank 2, the matrix $\mathbf{A}_{1}^{\omega}$ has 0's on the diagonal and \pm 1 elsewhere. Hence it may be viewed as the Seidel matrix of a graph $\Gamma$, where the edges of $\Gamma$ are precisely the edges of $K_{n}$ that have weight -1 . The weight therefore represents a two-graph, and it is coherent precisely when the two-graph is regular. It is clear that the minimal polynomial of $\mathbf{A}_{1}^{\omega}$ is quadratic, since

$$
\left(\mathbf{A}_{1}^{\omega}\right)^{2}=\beta_{11}^{0} \mathbf{A}_{0}^{\omega}+\beta_{11}^{1} \mathbf{A}_{1}^{\omega}
$$

and $\mathbf{A}_{0}^{\omega}=\mathbf{I}$.

To get a combinatorial description of the $\beta_{i j}^{k}$, we return to the notion of a triangle $(x, y, z)$ of type $(i, j, k)$ recalling that this means $x, y, z \in X,(x, y) \in f_{i},(y, z) \in$ $f_{j}$, and $(x, z) \in f_{k}$. The weight of the triangle is simply the product of the weights on the three edges:

$$
\delta \omega(x, y, z)=\omega(x, y) \omega(y, z) \omega(z, x) .
$$

Now, for $(x, z) \in f_{k}$, the $(x, z)$ entry of the product (2) gives

$$
\sum_{y} \mathbf{A}_{i}^{\omega}(x, y) \mathbf{A}_{j}^{\omega}(y, z)=\beta_{i j}^{k} \mathbf{A}_{k}^{\omega}(x, z) .
$$


The summands on the left are nonzero only for those $y$ for which $(x, y, z)$ has type $(i, j, k)$, that is for $y \in f_{i}(x) \cap f_{j}^{T}(z)$. Thus

$$
\begin{aligned}
\sum \omega(x, y) \omega(y, z) & =\beta_{i j}^{k} \omega(x, z) \text { which implies } \\
\sum \delta \omega(x, y, z) & =\beta_{i j}^{k}
\end{aligned}
$$

where sums are over all $y$ such that $(x, y, z)$ has type $(i, j, k)$. Hence $\beta_{i j}^{k}$ is the sum of the weights of all such triangles. We now see that a weight is coherent on the $\mathrm{CC}$ if and only if this sum is independent of the choice of vertices $x$ and $z$ in the graph of $f_{k}$.

Separating the triangles of weight 1 from those of weight -1 gives a notion of regularity that extends that of regular two-graphs. Given $(x, z) \in f_{k}$, set $\beta_{i j}^{k}(x, z, \alpha)$ equal to the number of triangles $(x, y, z)$ of type $(i, j, k)$ and weight $\alpha$.

Definition 4 A weight with values in $U$ is regular on a CC $\mathcal{X}$ if $\beta_{i j}^{k}(x, z, \alpha)$ depends only on $i, j, k$, and $\alpha$ and not on the choice of vertices in $\Gamma_{k}$.

Observe that the total number of such triangles is $p_{i j}^{k}$, hence

$$
p_{i j}^{k}=\sum_{\alpha} \beta_{i j}^{k}(x, z, \alpha)=\beta_{i j}^{k}(x, z, 1)+\beta_{i j}^{k}(x, z,-1)
$$

and

$$
\beta_{i j}^{k}=\sum_{\alpha} \alpha \beta_{i j}^{k}(x, z, \alpha)=\beta_{i j}^{k}(x, z, 1)-\beta_{i j}^{k}(x, z,-1) .
$$

Proposition 1 A coherent weight with values $\{ \pm 1\}$ on a $C C \mathcal{X}$ is regular on $\mathcal{X}$.

Proof We can solve (3) and (4) for $\beta_{i j}^{k}(x, z, 1)$ and $\beta_{i j}^{k}(x, z,-1)$ in terms of $p_{i j}^{k}$ and $\beta_{i j}^{k}$, both of which are independent of $x$ and $z$.

\section{Coherent closures of regular weights}

For this section, we let $\omega$ be a weight with values \pm 1 , regular on a $\mathrm{CC} \mathcal{X}$, with notation as in Sect. 2. We investigate the CCL of $\mathcal{A}^{\omega}$, and find necessary conditions for this CCL to have minimal rank. It is immediate that the CCL of $\mathcal{A}^{\omega}$ under o must contain $\mathcal{A}$. Now let $\mathcal{B}$ be the algebra generated by the union of $\mathcal{A}$ and $\mathcal{A}^{\omega}$ or equivalently, as we shall see below, by the set $\left\{\mathbf{A}_{i}^{\omega}(\alpha)\right\}_{i=0, \ldots, \alpha= \pm 1}$, where the $(x, y)$ entry of $\mathbf{A}_{i}^{\omega}(\alpha)$ is defined to be 1 if $\mathbf{A}_{i}^{\omega}(x, y)=\alpha$, and 0 otherwise. $\mathcal{B}$ is an algebra under ordinary multiplication, but is not in general a coherent algebra. $\operatorname{Put} \mathcal{C}:=\operatorname{CCL}(\mathcal{B})$. Naturally $\mathcal{C}$ may be non-symmetric or non-commutative. Figure 1 shows the inclusions as matrix algebras.

The angled brackets in the diagram indicate the algebra generated, under ordinary matrix multiplication over $\mathbb{C}$, by the indicated set. Determining conditions under which 
Fig. 1 Coherent algebra inclusion (figure produced by $\mathrm{PiCTeX})$

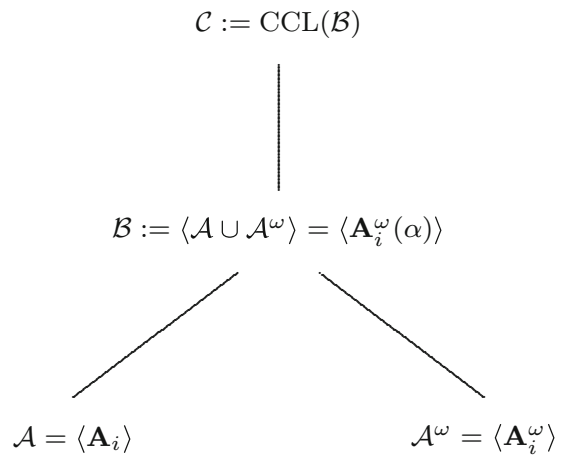

$\mathcal{B}$ and $\mathcal{C}$ coincide is a main goal of this work. The case in which $\mathcal{A}$ and $\mathcal{A}^{\omega}$ coincide is precisely the situation where $\omega$ is termed a trivial regular weight.

Lemma $1\left\langle\mathcal{A} \cup \mathcal{A}^{\omega}\right\rangle=\left\langle\mathbf{A}_{i}^{\omega}(\alpha)\right\rangle_{i=0, \ldots r, \alpha= \pm 1}$.

Proof Since $\mathbf{A}_{i}=\mathbf{A}_{i}^{\omega}(1)+\mathbf{A}_{i}^{\omega}(-1)$ and $\mathbf{A}_{i}^{\omega}=\mathbf{A}_{i}^{\omega}(1)-\mathbf{A}_{i}^{\omega}(-1)$, the union $\mathcal{A} \cup \mathcal{A}^{\omega}$ is contained in the right hand side. The reverse inclusion is obtained by solving these pairs of equations to get

$$
\begin{aligned}
\mathbf{A}_{i}^{\omega}(1) & =\frac{1}{2}\left(\mathbf{A}_{i}+\mathbf{A}_{i}^{\omega}\right) \\
\mathbf{A}_{i}^{\omega}(-1) & =\frac{1}{2}\left(\mathbf{A}_{i}-\mathbf{A}_{i}^{\omega}\right) .
\end{aligned}
$$

Notation We will henceforth use $\mathbf{A}_{i}^{+}$and $\mathbf{A}_{i}^{-}$to denote $\mathbf{A}_{i}^{\omega}(1)$ and $\mathbf{A}_{i}^{\omega}(-1)$ respectively whenever $\omega$ is clear from the context.

Lemma $2 \operatorname{CCL}\left(\mathcal{A}^{\omega}\right)=\operatorname{CCL}(\mathcal{B})$.

Proof Clearly, $\operatorname{CCL}\left(\mathcal{A}^{\omega}\right) \leq \operatorname{CCL}(\mathcal{B})$. On the other hand, $\operatorname{CCL}\left(\mathcal{A}^{\omega}\right)$, must contain

$$
\mathbf{A}_{i}^{\omega} \circ \mathbf{A}_{i}^{\omega}=\mathbf{A}_{i}
$$

so it contains both $\mathcal{A}$ and $\mathcal{A}^{\omega}$, hence $\mathcal{B}$. We conclude $\operatorname{CCL}\left(\mathcal{A}^{\omega}\right) \geq \operatorname{CCL}(\mathcal{B})$.

Lemma 3 Let $\left(X,\left\{g_{j}\right\}\right)$ be the CC underlying $C C L\left(\left\{\mathbf{A}_{i}^{\omega}\right\}\right)$. Then $\left(X,\left\{f_{i}\right\}\right)$ is a fusion of $\left(X,\left\{g_{j}\right\}\right)$.

Proof Since $\mathcal{A} \leq \mathcal{C}$ as coherent algebras, the associated CCs must be related by fusion [13].

It should be noted that the rank of the CCL of a regular weight is not preserved by switching. That is, two weights that are switching equivalent will typically not 
Fig. 2 Fission and fusion of weighted configurations

$$
\begin{array}{lc}
\mathcal{C}:=\left\langle\mathbf{C}_{0}, \mathbf{C}_{1}, \ldots, \mathbf{C}_{R}\right\rangle \stackrel{\circ \omega}{\longrightarrow} \quad \mathcal{C}^{\omega}:=\left\langle\mathbf{C}_{0}^{\omega}, \ldots, \mathbf{C}_{R}^{\omega}\right\rangle \\
\quad \text { fission }\left.\uparrow\right|_{\text {fusion }} & \text { fission }\left.\uparrow\right|_{\downarrow} \text { fusion } \\
\mathcal{A}:=\left\langle\mathbf{A}_{0}, \mathbf{A}_{1}, \ldots, \mathbf{A}_{r}\right\rangle \stackrel{\circ \omega}{\longrightarrow} \mathcal{A}^{\omega}:=\left\langle\mathbf{A}_{0}^{\omega}, \mathbf{A}_{1}^{\omega}, \ldots, \mathbf{A}_{r}^{\omega}\right\rangle
\end{array}
$$

have CCLs of the same rank. As weights, like two-graphs, are considered unique up to switching, the determination of whether a given weight has minimal CCL may in practice require analysis of the entire switching class. Conditions on the parameters, of course, apply to the whole class.

\section{Fusions of regular weights}

Let $\mathcal{C}$ be the CCL of a CC with coherent algebra $\mathcal{A}$ and regular weight $\omega$. Since $\omega$ is constant on $\mathbf{A}_{i}^{+}$and $\mathbf{A}_{i}^{-}$for all $i$, and these generate $\mathcal{B}, \omega$ is therefore constant on the relations of $\mathcal{C}$, a fission of $\mathcal{B}$. Setting $\mathbf{C}_{i}^{\omega}:=\omega \circ \mathbf{C}_{i}$, we obtain a trivial regular weight on $\mathcal{C}$.

On the other hand, we may recover $\mathcal{A}$ and $\mathcal{A}^{\omega}$ given $\mathcal{C}$ with $\mathbf{C}_{i}^{\omega}=\alpha_{i} \mathbf{C}_{i}, \alpha_{i} \in\{ \pm 1\}$, by applying the same fusion to $\mathcal{C}$ and $\mathcal{C}^{\omega}$. Here the merging is done according to a partition $\pi$ of the index set. Summations are over the indices in one part of the partition, so that

$$
\mathbf{A}_{j}:=\sum_{i \in \pi_{j}} \mathbf{C}_{i}
$$

and

$$
\mathbf{A}_{j}^{\omega}:=\omega \circ \sum_{i \in \pi_{j}} \mathbf{C}_{i}=\sum_{i \in \pi_{j}} \omega \circ \mathbf{C}_{i}=\sum_{i \in \pi_{j}} \mathbf{C}_{i}^{\omega} .
$$

In Fig. 2, it is important to note that $\mathcal{C}^{\omega}$ is a coherent algebra (and is equal to $\mathcal{C}$ ) while $\mathcal{A}^{\omega}$ is not (unless $\omega$ is trivial on $\mathcal{A}$ ).

Beginning with $\mathcal{A}$ and $\omega$, it is always possible to complete the diagram: the Schur idempotents for $\mathcal{C}$ are determined by the stabilization process, while the standard basis of $\mathcal{C}^{\omega}$ is given by $\left\{\omega \circ \mathbf{C}_{i}\right\}$.

Beginning with $\mathcal{C}$, we may or may not be able to complete the diagram. Various trivial weights on $\mathcal{C}$ may be investigated, various mergings may be tested for coherence, and only if the induced weight on the fusion turns out to be coherent can the diagram be completed.

\subsection{Parameter conditions for coherent fusions}

In this section we consider the conditions under which a CC of rank $R+1$ has a trivial weight supporting fusion to a regular weight on a $\mathrm{CC}$ of rank $r+1$. There are two 
requirements here: (1) that some partition affords a fusion scheme of rank $r+1$; (2) that the weight induced by the trivial weight and the fusion be coherent on the rank $r+1$ configuration. We determine parameter conditions for this situation.

Let $\mathcal{X}$ be a CC of rank $R+1$ with structure constants $\left\{s_{i j}^{k}\right\}$. A fusion of $\mathcal{X}$ to a rank $r+1 \mathrm{CC}$ is coherent if $\mathcal{I}:=\{0,1, \ldots, R\}$ is partitioned into $\mathcal{J}_{0}, \mathcal{J}_{1}, \mathcal{J}_{2}, \ldots, \mathcal{J}_{r}$ and

$$
\sum_{i \in \mathcal{J}_{s}} \sum_{j \in \mathcal{J}_{t}} s_{i j}^{k_{1}}=\sum_{i \in \mathcal{J}_{s}} \sum_{j \in \mathcal{J}_{t}} s_{i j}^{k_{2}} \forall s, t, u \in\{0,1, \ldots, r\} \text { and } \forall k_{1}, k_{2} \in \mathcal{J}_{u} \text {. }
$$

In this case, the parameters of the fusion scheme are given by

$$
p_{s t}^{u}:=\sum_{i \in \mathcal{J}_{s}} \sum_{j \in \mathcal{J}_{t}} s_{i j}^{k}
$$

for any $k \in \mathcal{J}_{u}$.

Suppose now that $\mathcal{X}$ has a coherent fusion to a CC $\mathcal{Y}=\left(X,\left\{f_{i}\right\}_{i \in \mathcal{J}}\right)$ of rank $r+1$, where $\mathcal{J}=\{0,1, \ldots, r\}$. Define a trivial weight on $\mathcal{X}$ by setting $\mathbf{A}_{i}^{\omega}:=\alpha_{i} \mathbf{A}_{i}$, where $\alpha_{i} \in\{ \pm 1\}$ for all $i \in \mathcal{I}$ and $\alpha_{0}=1$. Applying the fusion to our weight we see that the induced weight on $\mathcal{Y}$ is coherent provided the structure constants $\beta_{i j}^{k}$, are well defined. That is, for all $s, t, u \in \mathcal{J}$ and for all $k_{1}, k_{2} \in \mathcal{J}_{u}$,

$$
\alpha_{k_{1}} \sum_{i \in \mathcal{J}_{s}} \sum_{j \in \mathcal{J}_{t}} \alpha_{i} \alpha_{j} s_{i j}^{k_{1}}=\alpha_{k_{2}} \sum_{i \in \mathcal{J}_{s}} \sum_{j \in \mathcal{J}_{t}} \alpha_{i} \alpha_{j} s_{i j}^{k_{2}}
$$

In this case, $\mathcal{Y}$ has parameters given by

$$
\beta_{s t}^{u}=\alpha_{k} \sum_{i \in \mathcal{J}_{s}} \sum_{j \in \mathcal{J}_{t}} \alpha_{i} \alpha_{j} s_{i j}^{k}
$$

for each $k \in \mathcal{J}_{u}$.

Note: Condition (7) holds if $s_{i j}^{k_{1}}=s_{i j}^{k_{2}}$ for all $i \in \mathcal{J}_{s}, j \in \mathcal{J}_{t}, k_{1}, k_{2} \in \mathcal{J}_{u}$ but other solutions are possible. In particular, $\alpha_{k_{1}}=\alpha_{k_{2}}$ need not be the case in general.

\section{Examples}

\subsection{The 4-cube}

The 4-cube is a diameter $4 \mathrm{DRG}$, or rank 5 association scheme with vertex set $\mathbb{F}_{2}^{4}$. The relations are given by the distance graphs where the distance between two 4-tuples is the number of indices in which they differ.

Applying the fusion $\mathbf{A}_{0}, \mathbf{A}_{3}+\mathbf{A}_{4}, \mathbf{A}_{1}+\mathbf{A}_{2}$ to this scheme gives the Clebsch graph, or $\operatorname{SRG}(16,5,0,2)$. This graph may be described in the following way: the vertices are the 16 even-cardinality subsets of $\{1,2, \ldots, 5\}$. Two vertices are adjacent when their symmetric difference has cardinality 4 . 
Now, apply the same fusion to a trivial weight on the 4-cube to get a regular weight on the Clebsch graph. We merge classes 3 and 4, 1 and 2, as before, but this time put a negative weight on classes 2 and 4 . Thus our weighted adjacency matrices are

$$
\text { I, } \mathbf{A}_{3}-\mathbf{A}_{4}, \mathbf{A}_{1}-\mathbf{A}_{2} \text {. }
$$

This weight is regular, and the CCL of its adjacency algebra is the (dimension 5) coherent algebra of the 4-cube.

Observe that we may identify $\mathbb{F}_{2}^{4}$ with subsets of $\{1,2,3,4\}$, and that these are in one-to-one correspondence with the even subsets of $\{1,2,3,4,5\}$. (Each odd cardinality subset $S$ of the former corresponds to $S \cup\{5\}$.) Now, since the 4-tuples at distance 3 or 4 have symmetric difference of cardinality 4 , we see that $\mathbf{A}_{3}+\mathbf{A}_{4}$ gives the Clebsch graph, and $\mathbf{A}_{1}+\mathbf{A}_{2}$ its complement. The edges are weighted so that the CCL refines the classes according to distance and we recover the 4-cube.

\section{2 $\operatorname{SRG}(126,25,8,4)$}

This example is from the Johnson scheme $J(9,4)$. The rank 5 scheme also arises as a distance regular graph on 126 vertices, with valencies 1, 5, 20, 40, 60 and distance array $\{5,4,4,3 ; 1,1,2,2\}$ [5]. It is known as the Odd graph on 9 points; merging classes 1 and 2 gives SRG(126, 25, 8, 4). The Odd graph is Q-polynomial with ordering $0,4,1,3,2$. Lastly, this scheme may be obtained from a rank 3 group action of the alternating group $A_{10}$.

The vertices are the $\left(\begin{array}{l}9 \\ 4\end{array}\right)$ 4-sets from the set $\{1,2, \ldots, 9\}$, with adjacency when the two sets are disjoint. Clearly, the Johnson scheme $J(9,4)$ is related, with the same vertex set and relations determined by the intersection sizes. Indeed, this Johnson scheme has two P-polynomial orderings, the standard one: 0, 1, 2, 3, 4 and 0, 4, 1, 3, 2. We see that the Odd graph is the DRG determined by the second of these orderings.

The intersection matrices, $\mathbf{M}_{1}:=\left(p_{i 1}^{k}\right)$ and $\mathbf{M}_{2}:=\left(p_{i 2}^{k}\right)$ are below.

$$
\left(\begin{array}{rrr} 
& 1 & \\
25 & 8 & 4 \\
& 16 & 21
\end{array}\right) \quad\left(\begin{array}{rrr} 
& & 1 \\
16 & 21 \\
100 & 84 & 78
\end{array}\right)
$$

The weighted intersection matrices, $\mathbf{M}_{1}^{\omega}:=\left(\beta_{i 1}^{k}\right)$ and $\mathbf{M}_{2}^{\omega}:=\left(\beta_{i 2}^{k}\right)$ are

$$
\left(\begin{array}{rrr} 
& 1 & \\
25 & -8 & -4 \\
& -16 & -3
\end{array}\right) \quad\left(\begin{array}{rrr} 
& & 1 \\
& -16 & -3 \\
100 & -12 & -6
\end{array}\right) .
$$

A regular weight with the same parameters is obtained through the rank 3 action of $A_{10}$ on bisections - unordered pairs of 5-sets from $\{1,2, \ldots, 10\}$ [22]. Using techniques developed by Higman in [14], a transitive monomial representation may be constructed with centralizer algebra $\mathcal{A}^{\omega}$. The fusion described above is therefore another construction of a known regular weight. 
Observe that for certain triples $(i, j, k), \beta_{i j}^{k}=-\beta_{i j}^{k}(-1)=-p_{i j}^{k}$. All triangles of these types have weight -1 , as $\beta_{i j}^{k}(1)=0$. But this weight is not trivial. The eigenvalues and multiplicities are:

\begin{tabular}{|c|c|c|c|c|c|}
\hline & I $\begin{array}{ll}\mathbf{A}_{1} & \mathbf{A}_{2}\end{array}$ & & & I $\quad \mathbf{A}_{1}^{\omega} \quad \mathbf{A}_{2}^{\omega}$ & \\
\hline$\zeta_{1}$ & 125100 & 1 & $\zeta_{1}$ & $5-10$ & 42 \\
\hline$\zeta_{2}$ & $1-3 \quad 2$ & 90 & $\zeta_{2}$ & $\begin{array}{ll}1 & -1\end{array}$ & 75 \\
\hline$\zeta_{3}$ & $7-8$ & 35 & $\zeta_{3}$ & $1-15-20$ & 9 \\
\hline
\end{tabular}

\subsection{Folded 9-cube}

This example on 256 vertices comes from the folded 9-cube, also known as a generalized Odd graph. The rank 5 scheme is Q-polynomial with two distinct orderings. (These DRGs are described in [5, Sects. 6.3 and 6.4] and have distance array $[9,8,7,6 ; 1,2,3,4]$.) The folded $n$-cube has antipodal pairs of vertices in the $n$-cube as its vertices, with two such pairs adjacent if the possible distances between them are 1 and $n-1$.

The intersection matrices, eigenvalues and multiplicities are given below. Observe that this is a non-trivial weight on $\operatorname{SRG}(256,45,16,6)$ obtained by merging classes 1 and 2. We denote the fusion to the weight by $\left\{1^{-} 2^{+}\right\}\left\{3^{-} 4^{+}\right\}$, meaning that $\mathbf{A}_{1}^{\omega}$ $=-\mathbf{B}_{1}+\mathbf{B}_{2}$ and $\mathbf{A}_{2}^{\omega}=-\mathbf{B}_{3}+\mathbf{B}_{4}$.

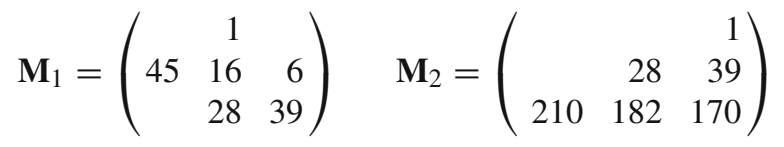

$$
\begin{aligned}
& \mathbf{M}_{1}^{\omega}=\left(\begin{array}{rrr}
1 & & \\
45 & -16 & -6 \\
& -28 & -9
\end{array}\right) \quad \mathbf{M}_{2}^{\omega}=\left(\begin{array}{rrr} 
& & 1 \\
& -28 & -9 \\
210 & -42 & -10
\end{array}\right)
\end{aligned}
$$

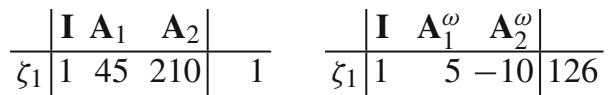

$$
\begin{aligned}
& \begin{array}{l|rrr|rr|rrr|r}
\zeta_{2} & 1 & 13 & -14 & 45 & \zeta_{2} & 1 & -27 & -42 & 10 \\
\zeta_{3} & 1 & -3 & 2 & 210 & \zeta_{3} & 1 & -3 & 14 & 120
\end{array}
\end{aligned}
$$

There are two P-polynomial orderings for this scheme, the standard one and $\{2,4,3,1\}$, thus we obtain the same SRG and weight by merging classes 1 and 4 in the halved 9-cube, $\operatorname{DRG}(36,21,10,3 ; 1,6,15,28)$. This regular weight is new, as far as the authors are aware, and in particular does not arise through a transitive monomial representation of any ATLAS groups [8,23].

\section{Rank 2 case}

Two questions regarding regular two-graphs and SRGs are answered in the propositions quoted below, due to Don Taylor. The first answers the question: When does the 
Seidel matrix of an SRG give a regular two-graph? The answer quite naturally gives conditions on the SRG parameters.

Proposition 2 ([28, Prop. 2.4.]) Let $\mathbf{A}_{1}$ be the (0, 1)-adjacency matrix of $\operatorname{SRG}(n, k$, $\lambda, \mu)$ and set $\mathbf{A}_{2}=\mathbf{J}-\mathbf{A}_{1}-\mathbf{I}$. Then $\mathbf{A}_{2}-\mathbf{A}_{1}$ is the matrix of a regular two-graph if and only if $n=2(2 k-\lambda-\mu)$.

The second question is, in a sense, the reverse of the first one: When does the switching class of a regular two-graph contain an SRG? Here the answer takes the form of restrictions on the regular two-graph and on the SRG parameters. Note that the notation of Sect. 2.4, particularly the $\beta_{i j}^{k}$, applies to the regular two-graphs, as we may view them as regular weights on complete graphs. The result below is strengthened in [5, Theorem 1.5.6 and addendum].

Proposition 3 ([28, Prop. 3.6.]) If the switching class of a regular two-graph contains an $S R G$, then either $\beta_{11}^{1}=0$ and $n-1$ is the sum of two squares; or the eigenvalues of $\mathbf{A}_{1}$ are odd integers $\rho_{1} \geq \rho_{2}$ and the srg parameters satisfy (I) or (II) below, with $\sigma_{1}=\frac{1}{2}\left(\rho_{1}-1\right), \sigma_{2}=-\frac{1}{2}\left(\rho_{2}+1\right), n=2\left(\sigma_{1}+\sigma_{2}+2 \sigma_{1} \sigma_{2}+1\right), \beta_{11}^{1}=2\left(\sigma_{1}-\sigma_{2}\right)$.

$$
\begin{aligned}
k & =\left(2 \sigma_{1}+1\right) \sigma_{2} & & p_{11}^{0}=\left(\sigma_{1}+1\right)\left(2 \sigma_{2}+1\right) \\
\text { (I) } \quad \lambda & =\left(\sigma_{1}+1\right)\left(\sigma_{2}-1\right) & \text { (II) } & p_{11}^{1}=\left(\sigma_{1}+2\right) \sigma_{2} \\
\mu & =\sigma_{1} \sigma_{1} & & p_{11}^{2}=\left(\sigma_{1}+1\right)\left(\sigma_{2}+1\right)
\end{aligned}
$$

For example, the lattice graph $L_{2}(4)$ has SRG parameters $(16,6,2,2)$. We see this satisfies (II) above with $\sigma_{1}=1, \sigma_{2}=2, \rho_{1}=3, \rho_{2}=-5$, thus we have a regular two-graph represented by $\mathbf{J}-\mathbf{I}-2 \mathbf{A}_{1}=\mathbf{A}_{2}-\mathbf{A}_{1}$.

To generalize both of these propositions, we rephrase in light of the fact that a regular two-graph is a regular weight on a rank $2 \mathrm{CC}$ (i.e., the complete graph). In the first case, we observe that the Seidel matrix of an SRG is $S=-\mathbf{A}_{1}+\mathbf{A}_{2}$ in our notation, where $\mathbf{A}_{i}$ has $(0,1)$ entries. Hence we may view $S$ as a (trivial) weight on the SRG, with (-) weight on all edges of the graph and (+) on all edges of the complement. When $S$ determines a regular two-graph, the weight has a fusion to a regular weight on $K_{n}$. That is, the fusion $\mathbf{A}_{1}+\mathbf{A}_{2}$ gives $K_{n}$, the corresponding fusion applied to the weight is $\omega-\mathbf{I}=-\mathbf{A}_{1}+\mathbf{A}_{2}$, and this weight is regular. To generalize the question that Proposition 2 answers we therefore rephrase it as:

When does a trivial weight on a rank $3 \mathrm{CC}$ have a fusion to a nontrivial regular weight on a rank $2 \mathrm{CC}$ ?

The objects of interest are therefore regular weights obtained through fusions of trivial weights on higher rank CCs. In Sect. 8 we treat the rank 4 case. The distance-regular, rank 5 schemes have been analysed by the author, with regard to fusion and closure of regular weights. This forms part of a work in progress on the rank 5 case.

In a similar way, we may generalize Proposition 3 by rephrasing the question to:

When is the CCL of a rank 2 regular weight equal to a rank $3 \mathrm{CC}$ ? 
(More precisely, when does some weight in the switching class of a regular rank 2 weight have minimal coherent closure?) Here, we are interested in the case $\mathcal{B}=\mathcal{C}$ in the notation of Sect. 3 .

Generally, the minimal rank of the CCL is $1+2(r-1)=2 r-1$ when $\mathcal{A}$ has rank $r$. However, it may happen that $\mathbf{A}_{i}^{\omega}(\alpha)=0$ for some $i$ and some $\alpha$ hence lower rank than $2 r-1$ is possible. We use the term minimal rank to mean $\left|\left\{\mathbf{A}_{i}^{\omega}(\alpha) \mid \mathbf{A}_{i}^{\omega}(\alpha) \neq 0\right\}\right|$. A quick example: suppose we have an SRG with edges weighted \pm 1 but all nonedges weighted -1 . In that case, $\mathbf{A}_{2}^{\omega}(1)=0, \mathcal{B}$ is generated by the four matrices $\mathbf{A}_{0}^{\omega}=\mathbf{I}, \mathbf{A}_{1}^{+}, \mathbf{A}_{1}^{-}$, and $\mathbf{A}_{2}^{-}$so the minimal rank of the CCL is 4 .

\section{A family of rank 6 closures}

We begin with an example of a rank 6 association scheme on 256 points_primitive, commutative, neither metric nor cometric-which is the CCL of a regular weight on $L_{2}(16)=\operatorname{SRG}(256,30,14,2)$. We then extend the construction to the family $L_{2}(n)$ whenever there exists a regular two-graph on $n$ vertices, and show that the CCL has rank 6 precisely when the two-graph has minimal CCL.

In [23] a construction is given for regular weights on the lattice graphs, $L_{2}(n)$ $=S R G\left(n^{2}, 2(n-1), n-2,2\right)$ from the tensor product of two regular two-graphs. Here, we obtain the regular weight from $\mathbf{T} \otimes^{2}$ where $\mathbf{T}$ is a matrix of regular two-graph $\tau$.

There is a unique regular two-graph on 16 points represented by the matrix

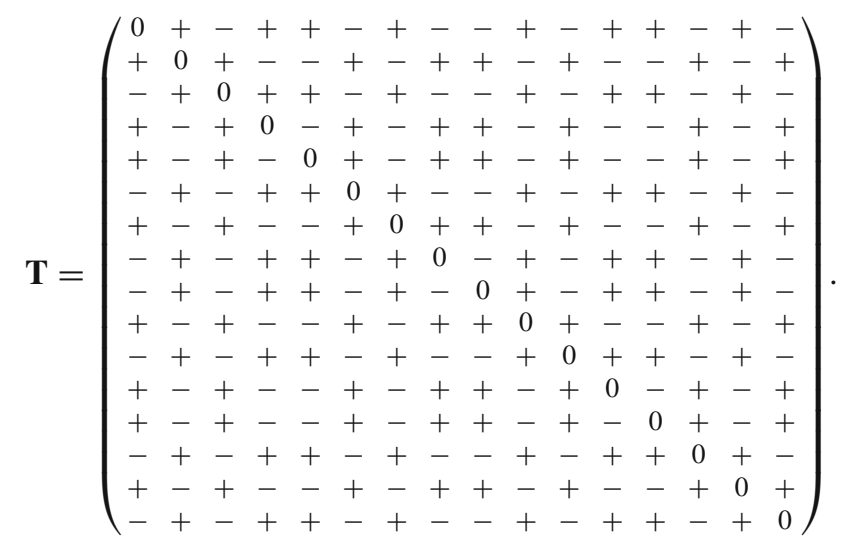

Set

$$
\begin{gathered}
\mathbf{A}_{1}:=\mathbf{I} \otimes(\mathbf{J}-\mathbf{I})+(\mathbf{J}-\mathbf{I}) \otimes \mathbf{I}, \quad \mathbf{A}_{2}:=(\mathbf{J}-\mathbf{I}) \otimes(\mathbf{J}-\mathbf{I}), \quad \mathbf{A}_{0}:=\mathbf{I} \otimes \mathbf{I}, \\
\text { and } \omega:=(\mathbf{I}+\mathbf{T}) \otimes(\mathbf{I}+\mathbf{T}) .
\end{gathered}
$$

The products $\mathbf{A}_{i}^{\omega} \mathbf{A}_{j}^{\omega}$ result in weighted intersection matrices

$$
\mathbf{M}_{1}^{\omega}=\left(\begin{array}{rrr} 
& 1 & \\
30 & 2 & 2 \\
& 15 & 4
\end{array}\right) \quad \mathbf{M}_{2}^{\omega}=\left(\begin{array}{rrr} 
& 15 & 4 \\
225 & 30 & 4
\end{array}\right) .
$$


Clearly,

$$
\begin{aligned}
& \mathbf{A}_{1}^{-}=\mathbf{I} \otimes \mathbf{T}^{-}+\mathbf{T}^{-} \otimes \mathbf{I}, \\
& \mathbf{A}_{1}^{+}=\mathbf{I} \otimes \mathbf{T}^{+}+\mathbf{T}^{+} \otimes \mathbf{I}, \\
& \mathbf{A}_{2}^{-}=\mathbf{T}^{-} \otimes \mathbf{T}^{+}+\mathbf{T}^{+} \otimes \mathbf{T}^{-}, \\
& \mathbf{A}_{2}^{+}=\mathbf{T}^{-} \otimes \mathbf{T}^{-}+\mathbf{T}^{+} \otimes \mathbf{T}^{+},
\end{aligned}
$$

and the minimal rank of the CCL is therefore 5. In fact, $\mathbf{A}_{2}^{\omega}(1)$ splits in the CCL, and we obtain a rank 6 scheme with relations:

\section{$\mathbf{I}, \mathbf{I} \otimes \mathbf{T}^{-}+\mathbf{T}^{-} \otimes \mathbf{I}, \mathbf{I} \otimes \mathbf{T}^{+}+\mathbf{T}^{+} \otimes \mathbf{I}, \mathbf{T}^{-} \otimes \mathbf{T}^{+}+\mathbf{T}^{+} \otimes \mathbf{T}^{-}, \mathbf{T}^{-} \otimes \mathbf{T}^{-}, \mathbf{T}^{+} \otimes \mathbf{T}^{+}$.}

This construction is possible with any regular two-graph. Indeed, the regular weight on $L_{2}(n)$ obtained from a regular two-graph represented by the $(0, \pm 1)$ matrix $\mathbf{T}$ has intersection matrices

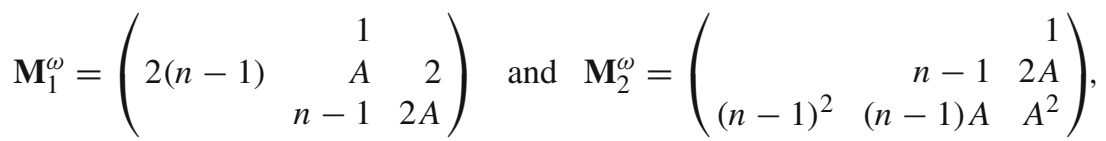

where $A$ is the two-graph parameter, that is, $\mathbf{T}^{2}=(n-1) \mathbf{I}+A \mathbf{T}$ [23].

Put $\omega:=(\mathbf{I}+\mathbf{T}) \otimes(\mathbf{I}+\mathbf{T})$ and $\mathbf{A}_{i}^{+}, \mathbf{A}_{i}^{-}$as above.

Proposition 4 A regular weight on $L_{2}(n)$ from a regular two-graph with matrix $\mathbf{T}$ has coherent closure of rank $\geq 6$, with equality if and only if $\mathbf{T}^{-}$is the matrix of a (possibly imprimitive) SRG.

Proof The product $\left(\mathbf{A}_{1}^{-}\right)^{2}$ is $\mathbf{I} \otimes\left(\mathbf{T}^{-}\right)^{2}+2 \mathbf{T}^{-} \otimes \mathbf{T}^{-}+\left(\mathbf{T}^{-}\right)^{2} \otimes \mathbf{I}$ which does not lie in $\operatorname{span}\left(\mathbf{I}, \mathbf{A}_{i}^{-}, \mathbf{A}_{i}^{+}\right)$. The middle term forces $\mathbf{A}_{2}^{+}$to split into $\mathbf{T}^{-} \otimes \mathbf{T}^{-}$and $\mathbf{T}^{+} \otimes \mathbf{T}^{+}$. This shows that the rank of the CCL is at least 6 . For equality, we must have no further splitting, hence $\left(\mathbf{T}^{-}\right)^{2}$ must lie in the span of $\mathbf{I}, \mathbf{T}^{-}$, and $\mathbf{T}^{+}$. But then $\mathbf{T}^{-}$is the matrix of an SRG.

Conversely, suppose that $\mathbf{T}^{-}$is the matrix of $\operatorname{SRG}(n, k, \lambda, \mu)$, and set $\mathbf{B}_{0}=\mathbf{I}, \mathbf{B}_{1}$ $=\mathbf{A}_{1}^{-}, \mathbf{B}_{2}=\mathbf{A}_{1}^{+}, \mathbf{B}_{3}=\mathbf{A}_{2}^{-}, \mathbf{B}_{4}=\mathbf{T}^{-} \otimes \mathbf{T}^{-}, \mathbf{B}_{5}=\mathbf{T}^{+} \otimes \mathbf{T}^{+}$. The products $\mathbf{B}_{i} \mathbf{B}_{j}$ can be worked out directly in terms of $n, k, \lambda, \mu, \bar{\lambda}:=n-2 k+\mu-2$, and $\bar{\mu}:=n-2 k+\lambda$, resulting in the intersection matrices below. Here $\sigma:=k-\lambda-1$ and $\rho:=k-\mu$.

$$
\mathbf{M}_{1}=\left(\begin{array}{cccccc} 
& 1 & & & & \\
2 k & \lambda & \mu & & 2 & \\
& \sigma & \rho & 1 & & \\
& k & \lambda+\rho & 2 \sigma & 2 \mu \\
& k & & \mu & 2 \lambda & \\
& & & \sigma & & 2 \rho
\end{array}\right), \quad \mathbf{M}_{2}=\left(\begin{array}{cccccc} 
& & 1 & & & \\
& \sigma & \rho & 1 & & \\
2 l & \bar{\mu} & \bar{\lambda} & & & 2 \\
& l & & \sigma+\bar{\lambda} & 2 \bar{\mu} & 2 \rho \\
& & & \rho & 2 \sigma & \\
& & l & \bar{\mu} & & 2 \bar{\lambda}
\end{array}\right),
$$




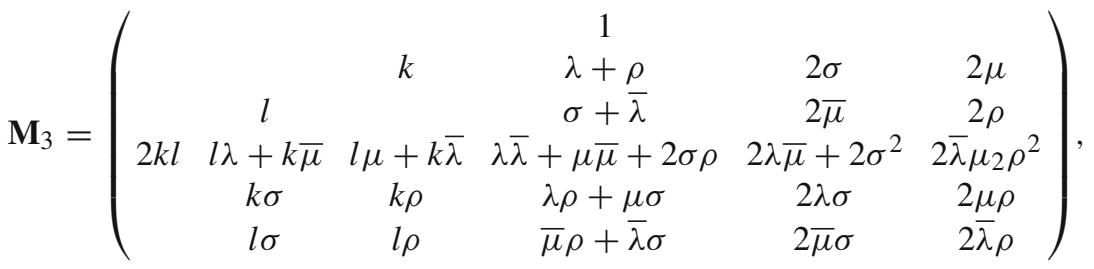

$$
\begin{aligned}
& \mathbf{M}_{4}=\left(\begin{array}{cccccc} 
& & & & & 1 \\
& k & & \mu & 2 \lambda & \\
& & & \rho & 2 \sigma & \\
& k \sigma & k \rho & \lambda \rho+\mu \sigma & 2 \lambda \sigma & 2 \mu \rho \\
k^{2} & k \lambda & k \mu & \lambda \mu & \lambda^{2} & \mu^{2} \\
& & & \sigma \rho & \sigma^{2} & \rho^{2}
\end{array}\right),
\end{aligned}
$$

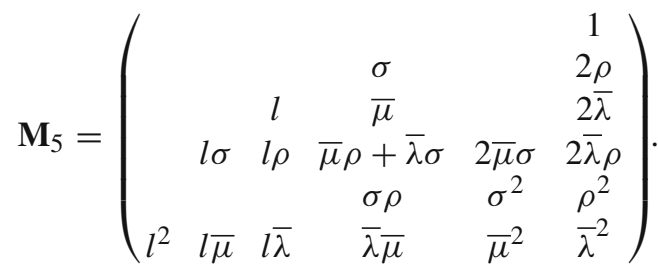

Note that the condition on $\tau$ is precisely that of Proposition 2, hence the SRG necessarily satisfies $n=2(2 k-\lambda-\mu)$, and we see that the minimal CCL for this family is achieved when $\tau$ itself has minimal CCL.

For the $n=16$ example, the rank 6 character-multiplicity table is

\begin{tabular}{l|rrrrrr|r} 
& $\mathbf{I}$ & $\mathbf{A}_{1}$ & $\mathbf{A}_{2}$ & $\mathbf{A}_{3}$ & $\mathbf{A}_{4}$ & $\mathbf{A}_{5}$ & $m_{i}$ \\
\hline$\zeta_{1}$ & 1 & 12 & 18 & 36 & 108 & 81 & 1 \\
$\zeta_{2}$ & 1 & 4 & 10 & -12 & -12 & 9 & 18 \\
$\zeta_{3}$ & 1 & 8 & 6 & 12 & 0 & -27 & 12 \\
$\zeta_{4}$ & 1 & -4 & 2 & 4 & -4 & 1 & 81 \\
$\zeta_{5}$ & 1 & 0 & -2 & -4 & 8 & -3 & 108 \\
$\zeta_{6}$ & 1 & 4 & -6 & 4 & -12 & 9 & 36
\end{tabular}

The fusion $\{12\}\{345\}$ with signs $\{-+\}\{+-+\}$ determines a regular weight on $\operatorname{SRG}(256,30,14,2)$. Notice, this can be seen from the character table as $\pm \mathbf{A}_{1}+\mathbf{A}_{2}$ and $\mathbf{A}_{3} \pm \mathbf{A}_{4}+\mathbf{A}_{5}$ all have three distinct eigenvalues.

\section{Rank 4 closures}

In this section, we investigate rank 4 CCLs, of regular $( \pm 1)$ weights on SRGs. As noted at the end of Sect. 6, a CCL of rank 4 may occur when the weight is constant on either $\mathbf{A}_{1}$ or $\mathbf{A}_{2}$. We show that such a scheme is necessarily imprimitive, with one relation a disjoint union of complete graphs.

The imprimitive rank 4 schemes were studied by Y. Chang and T. Huang in [7], using the term ISA to denote an imprimitive (symmetric) association scheme, excluding the 


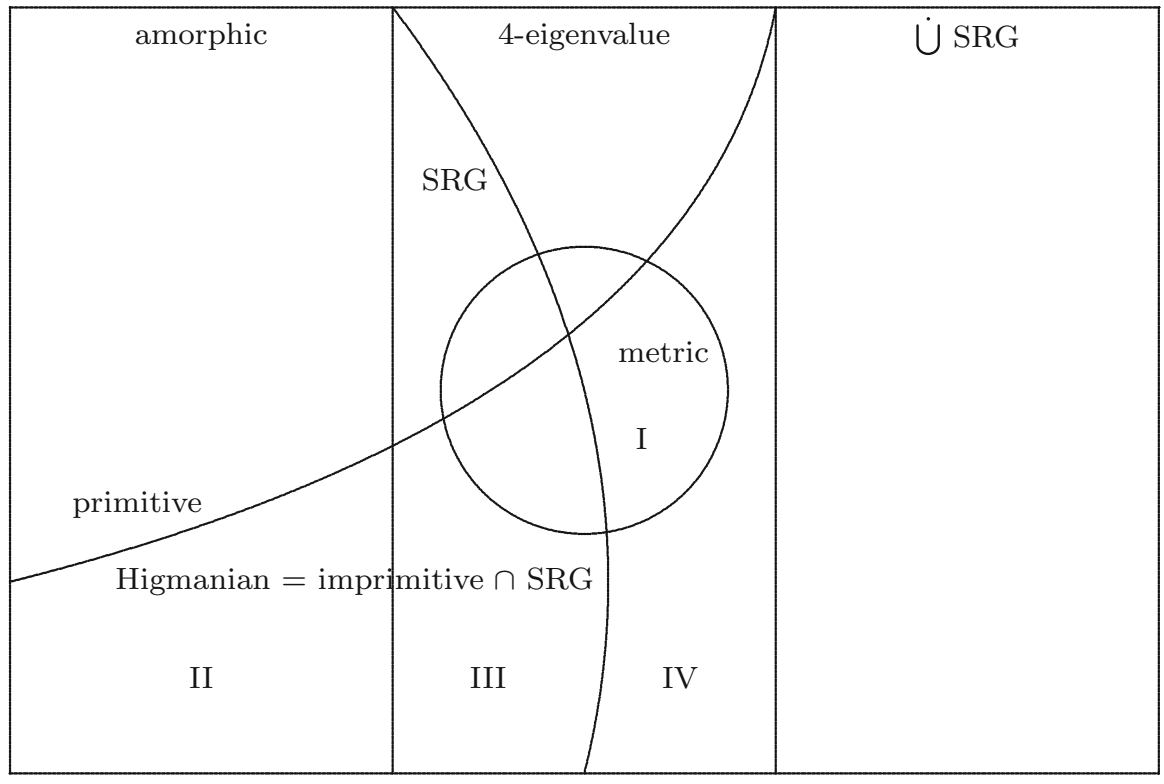

Fig. 3 Rank 4 association schemes (figure produced by PiCTeX)

"trivial" tensor products and wreath products of schemes. If at least one of the relations in such a scheme is an SRG, the SRG is called Higmanian. Here, we will call a rank 4 scheme Higmanian if it is imprimitive and has at least one SRG among its relations.

E. van Dam in [30] classified the rank 4 schemes as follows: (i) at least one of the $\mathbf{A}_{i}$ has four distinct eigenvalues; (ii) at least one of the relations is the disjoint union of (connected) SRGs with the same parameters; (iii) all three relations are SRGs, this latter group called the amorphic schemes. The schemes in group (i) are characterized in [30] and include the DRGs. The rank 4 CCLs we obtain are either amorphic (Higmanian with respect to two relations), Higmanian but not amorphic, or have two relations with four distinct eigenvalues. We use the following notations in Fig. 3.

amorphic: $\Gamma_{i}$ is strongly regular, possibly imprimitive, for all $i$.

4-eigenvalue: $\Gamma_{i}$ has 4 distinct eigenvalues, for at least one $i$.

U SRG: $\Gamma_{i}$ is a disjoint union of some (connected) SRGs having the same parameters, for at least one $i$.

SRG: $\Gamma_{i}$ is strongly regular (primitive) for at least one $i$.

metric: $\Gamma_{i}$ is distance-regular for at least one $i$.

Higmanian: Imprimitive SRG scheme.

primitive: $\Gamma_{i}$ is connected for all $i$.

I-IV: labels for the schemes which occur as CCLs.

Let $\mathcal{A}$ be the coherent algebra of a rank $r+1$ scheme with Schur idempotents $\mathbf{A}_{i}$. Let $\omega$ be a regular weight on $\mathcal{A}$ with values $\{ \pm 1\}$ and the usual notation $\mathcal{A}^{\omega}, \mathbf{A}_{i}^{\omega}=\omega \circ \mathbf{A}_{i}$. 
We know

$$
\mathbf{A}_{i} \mathbf{A}_{j}=\sum_{k} p_{i j}^{k} \mathbf{A}_{k} \quad \text { and } \quad \mathbf{A}_{i}^{\omega} \mathbf{A}_{j}^{\omega}=\sum_{k} \beta_{i j}^{k} \mathbf{A}_{k}^{\omega}
$$

Recall from Sect. 2 that $\mathbf{A}_{i}=\mathbf{A}_{i}^{+}+\mathbf{A}_{i}^{-}$and $\mathbf{A}_{i}^{\omega}=\mathbf{A}_{i}^{+}-\mathbf{A}_{i}^{-}$. Suppose that $\operatorname{CCL}\left(\mathcal{A}^{\omega}\right)$ is a rank 4 association scheme denoted $\mathcal{B}$, with structure constants $s_{i j}^{k}, 0 \leq$ $i, j, k \leq 3$. A rank 4 CCL requires that $\omega$ be all positive or all negative on either $\mathbf{A}_{1}$ or $\mathbf{A}_{2}$. That is, one of the $\mathbf{A}_{i}^{+}$or $\mathbf{A}_{i}^{-}$is zero. Let us assume for now that $\Gamma$ and $\omega$ are given by the fusion $\left\{1^{-} 2^{+}\right\}\left\{3^{+}\right\}$, so that $\mathbf{B}_{1}=\mathbf{A}_{1}^{-}, \mathbf{B}_{2}=\mathbf{A}_{1}^{+}, \mathbf{B}_{3}=\mathbf{A}_{2}^{+}$and $\mathbf{A}_{2}^{-}=0$.

We claim that $\mathcal{B}$ is symmetric. Indeed, all homogeneous CCs of rank up to 5 are commutative [13]. If $\mathcal{B}$ is not symmetric, then $\mathbf{B}_{1}^{T}=\mathbf{B}_{2}$ because $\mathbf{B}_{3}=\mathbf{A}_{2}$, which is symmetric by assumption. But then

$$
\left(\mathbf{A}_{1}^{\omega}\right)^{T}=\left(-\mathbf{B}_{1}+\mathbf{B}_{2}\right)^{T}=-\mathbf{B}_{2}+\mathbf{B}_{1}=-\mathbf{A}_{1}^{\omega}
$$

contradicting the fact that $\mathcal{A}^{\omega}$ is symmetric.

Substituting $\mathbf{A}_{1}=\mathbf{B}_{1}+\mathbf{B}_{2}, \mathbf{A}_{1}^{\omega}=-\mathbf{B}_{1}+\mathbf{B}_{2}, \mathbf{A}_{2}=\mathbf{A}_{2}^{\omega}=\mathbf{B}_{3}$ into (9), and equating coefficients of $\mathbf{B}_{i}$, we obtain:

$$
\begin{aligned}
& p_{11}^{1}=s_{11}^{1}+2 s_{12}^{1}+s_{22}^{1}=s_{11}^{2}+2 s_{12}^{2}+s_{22}^{2}, \\
& \beta_{11}^{1}=-s_{11}^{1}+2 s_{12}^{1}-s_{22}^{1}=s_{11}^{2}-2 s_{12}^{2}+s_{22}^{2}, \\
& p_{11}^{2}=s_{11}^{3}+2 s_{12}^{3}+s_{22}^{3}, \\
& \beta_{11}^{2}=s_{11}^{3}-2 s_{12}^{3}+s_{22}^{3}, \\
& p_{12}^{1}=s_{13}^{1}+s_{23}^{1}=s_{13}^{2}+s_{23}^{2}, \\
& \beta_{12}^{1}=s_{13}^{1}-s_{23}^{1}=-s_{13}^{2}+s_{23}^{2}, \\
& p_{12}^{2}=s_{13}^{3}+s_{23}^{3}, \\
& \beta_{12}^{2}=-s_{13}^{3}+s_{23}^{3}, \\
& p_{22}^{1}=s_{33}^{1}=s_{33}^{2}, \\
& \beta_{22}^{1}=-s_{33}^{1}=s_{33}^{2}, \\
& p_{22}^{2}=s_{33}^{3}, \\
& \beta_{22}^{2}=s_{33}^{3} .
\end{aligned}
$$

Equating two expressions for the sum of $p_{11}^{1}$ and $\beta_{11}^{1}$ from (10a) and (10b), and two expressions for their difference, gives

$$
\begin{aligned}
& 2 s_{12}^{1}=s_{11}^{2}+s_{22}^{2}, \\
& 2 s_{12}^{2}=s_{11}^{1}+s_{22}^{1} .
\end{aligned}
$$


Similarly, from (10e) and (10f):

$$
\begin{aligned}
& s_{13}^{1}=s_{32}^{2}, \\
& s_{23}^{1}=s_{13}^{2}
\end{aligned}
$$

which along with the standard identity $s_{i j}^{k} v_{k}=s_{k j}^{i} v_{i}$ proves that $v_{1}=v_{2}$ or $s_{23}^{1}=0$. Finally, from (10i) and (10j) we get

$$
s_{33}^{1}=s_{33}^{2}=0
$$

and this shows that any triple with two $\Gamma_{3}$ edges must have the third edge in $\Gamma_{3}$. Hence $\mathcal{B}$ is imprimitive with $\Gamma_{3}$ a disjoint union of cliques. We formulate our observations thus far as a lemma.

Lemma 4 Suppose $\mathcal{B}=C C L\left(\mathcal{A}^{\omega}\right)$ is a homogeneous $C C$ of rank 4 . Then $\mathcal{B}$ is a symmetric, imprimitive association scheme with $\Gamma_{3}$ a disjoint union of cliques and either $v_{1}=v_{2}$ or $s_{23}^{1}=0$.

Lemma 5 If $\operatorname{CCL}\left(\mathcal{A}^{\omega}\right)$ is a rank 4 association scheme and is metric, then the $P$ polynomial ordering is either $(1,2,3)$ or $(2,1,3)$.

The effect of this lemma is to rule out fusions other than $\{12\}\{3\}$ in the metric case, so that we may proceed to find rank 4 closures using this fixed notation for all cases.

Proof Clearly $\Gamma_{3}$ is not a DRG of diameter 3. The identities (10) through (13) are invariant under the transposition $(1,2)$ of indices provided we allow, as we may without loss of generality, $\omega$ to be negative on $\mathbf{B}_{2}$ and positive on $\mathbf{B}_{1}$. The cases we must consider are therefore (i) $\Gamma_{1}$ is a DRG with $\Gamma_{3}$ the distance 2 graph; (ii) $\Gamma_{1}$ is a DRG with $\Gamma_{3}$ the distance 3 graph.

To rule out (i), let $\Gamma_{1}$ be a DRG with distance 2 graph a union of cliques. Re-ordering the indices to match the P-polynomial ordering for $\mathcal{B}$, the fusion is now $\{13\}\{2\}$ and equation (13) gives $a_{2}=0$. Since $v_{1}=v_{3}, s_{13}^{3}=s_{33}^{1}=a_{3}$. Thus (11b) implies that $a_{1}=a_{3}$. Now employing $a_{i}+b_{i}+c_{i}=b_{0}$, we get

$$
c_{3}=b_{1}+1, \quad b_{2}=\frac{b_{0}\left(b_{1}+1\right)}{2 b_{1}+1}, \quad c_{2}=b_{0}-b_{2} .
$$

Because it is imprimitive, Smith's theorem ([5], Thm. 4.2.1) says that $\Gamma_{1}$ is bipartite and/or antipodal if $v_{1}>2$.

If $\Gamma_{1}$ is bipartite, the distance between any two vertices in the same half of the bipartition must be 2 , thus $\Gamma_{2}$ consists of exactly two cliques. In this case, $b_{1}=b_{0}-1$. But then

$$
b_{2}=\frac{b_{0}^{2}}{2 b_{0}-1} \text {, }
$$

an integer. However, $2 b_{0}-1$ is relatively prime to $b_{0}$, hence also to $b_{0}^{2}$ and thus $b_{0}$ must equal 1 . 
If $\Gamma_{1}$ is not bipartite but is antipodal, then $b_{2}=1$ and $c_{3}=b_{0}$. From (14) we again obtain $b_{0}=1$.

It remains to rule out $v_{1} \leq 2$. Let $t$ be the size of the $\Gamma_{2}$ cliques, so that $n$ is a multiple of $t$ and $n=1+v_{1}+v_{2}+v_{3} \leq t+4$. The possibilities for $(n, t)$ are $(8,4),(6,2)$, and $(4,2)$ but no DRGs with $v_{1}=v_{3}$ have these parameters.

From this point onward, we may assume the fusion is $\{12\}\{3\}$ and equations (10) through (13) hold. As $\Gamma$ is the complement of $\Gamma_{3}$, it has the imprimitive parameters SRG $(r t, t(r-1), t(r-2), t(r-1))$, where $t$ is the size of the cliques in $\Gamma_{3}$, and $r$ is the number of distinct cliques. We note in particular that $\beta_{22}^{2}=p_{22}^{2}=t-2$ and $p_{12}^{1}=t-1$.

\subsection{Metric case, $\mathbf{s}_{\mathbf{2 3}}^{\mathbf{1}} \neq \mathbf{0}$}

Returning to Lemma 4, suppose $s_{23}^{1} \neq 0$, which clearly includes the metric case. Since $v_{1}=v_{2}$ and thus $s_{12}^{1}=s_{11}^{2}$ and $s_{12}^{2}=s_{22}^{1}$, we use (11a) and (11b) to see that $s_{11}^{2}=s_{22}^{2}$ and $s_{11}^{1}=s_{22}^{1}$. The weight parameters therefore satisfy:

$$
\begin{aligned}
& \beta_{11}^{1}=2 s_{11}^{2}-2 s_{11}^{1}, \\
& \beta_{11}^{2}=2 s_{11}^{3}-2 s_{12}^{3}, \text { and } \\
& \beta_{12}^{1}=s_{13}^{1}-s_{23}^{1} .
\end{aligned}
$$

Suppose now that $\mathcal{B}$ is a metric scheme, i.e. that $\Gamma_{1}$ is a DRG with distance array $\left\{b_{0}, b_{1}, b_{2} ; c_{1}, c_{2}, c_{3}\right\}$. From above, $s_{33}^{1}=0$ gives $a_{3}=0$ which implies $c_{3}=b_{0}$. Further, $s_{11}^{3}$ must be 0 , hence $s_{22}^{3}=0$ and

$$
p_{12}^{1}=s_{13}^{2}=-\beta_{12}^{1} \text {. }
$$

From $v_{1}=v_{2}$, we obtain $c_{2}=b_{1}$, and since $s_{11}^{1}=s_{21}^{2}, a_{1}=a_{2}$. Now use the identity $a_{i}+b_{i}+c_{i}=b_{0}$ for $i=1,2$ to see that $b_{2}=c_{1}=1$. Finally, $v_{3}=1$, so the $\Gamma_{3}$-cliques have size 2 , and our distance array is $\left\{b_{0}, b_{1}, 1 ; 1, b_{1}, b_{0}\right\}$. The weighted intersection matrices and character-multiplicity table are as follows.

$$
\begin{aligned}
& \mathbf{M}_{1}^{\omega}=\left(\begin{array}{rrr}
1 & \\
2 b_{0} & \beta_{11}^{1} & -2 b_{0} \\
& -1 &
\end{array}\right) \quad \mathbf{M}_{2}^{\omega}=\left(\begin{array}{lll} 
& & 1 \\
1 & &
\end{array}\right) \\
& \begin{array}{l|lcr|l} 
& \mathbf{I} & \mathbf{A}_{1}^{\omega} & \mathbf{A}_{2}^{\omega} & \\
\hline \zeta_{1} & 1 & 0 & 1 & n / 2
\end{array}
\end{aligned}
$$

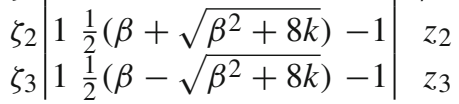

Note that either $\beta^{2}+8 k$ is a square, or $\beta=0$ and $z_{2}=z_{3}=n / 4$.

The DRGs obtained above are Taylor graphs ([5, Section 1.5]), in which $\Gamma_{1}$ is an antipodal double cover of $K_{b_{0}+1}$, or are antipodal and bipartite with $b_{1}=b_{0}-1$, and 
$\Gamma_{1}$ the complement of a $2 \times\left(b_{0}+1\right)$ grid. The weight parameter $\beta_{11}^{1}$ is $4 b_{1}-2 b_{0}+2$, which equals $p_{11}^{1}=2 b_{1}$ (i.e. $\lambda$ ) in the latter case and differs from $\lambda$ in the former.

The Taylor graphs are in one-to-one correspondence with the regular two-graphs, thus it is interesting that these should be precisely the DRGs that occur as minimal (rank 4) closures of weighted SRGs.

We have proven the following theorem. Parameters up to $n=100$ are given in Sect. 9.1, Table 2.

Theorem 1 Let $\Gamma_{1}$ be a distance-regular graph of diameter 3 with a fusion to an SRG $\Gamma$ and a nontrivial regular weight $\omega$, on $\Gamma$. Then $\Gamma=\Gamma_{1} \cup \Gamma_{2}, \Gamma_{1}$ is a Taylor graph with intersection array $\left\{b_{0}, b_{1}, 1 ; 1, b_{1}, b_{0}\right\}$, possibly the complement of a $2 \times\left(b_{0}+1\right)$ grid with $b_{1}=b_{0}-1$. Furthermore, $\Gamma^{\omega}$ has weighted intersection matrices as above, with $\beta_{11}^{1}=4 b_{1}-2 b_{0}+2$.

\subsection{General case, $\mathbf{s}_{\mathbf{2 3}}^{\mathbf{1}} \neq \mathbf{0}$}

We look next at the case in which $s_{23}^{1} \neq 0$ but $\mathcal{B}$ is not necessarily metric, we find that $\mathcal{B}$ is an imprimitive rank 4 scheme, the subject of [7], and belongs to the subclass with $v_{1}=v_{2}$. Intersection matrices and the character-multiplicity table for these rank 4 , imprimitive, (symmetric) association schemes, or rank 4 ISAs are given in that paper, but are presented here in our notation and ordering - note that in [7] it is $\Gamma_{1}$ that is a union of cliques. We give an independent verification.

Consider vertices $x, y$, and $z$ with $(x, y, z)$ forming a triangle of type $(1,3,2)$. The parameter $s_{13}^{1}$ counts vertices $w$, adjacent to $y$ in $\Gamma_{3}$, such that $w$ is adjacent to $x$ in $\Gamma_{1}$. On the other hand, $s_{23}^{2}$ counts all $v$, adjacent to $z$ in $\Gamma_{3}$ and adjacent to $x$ in $\Gamma_{2}$. In total, these count the neighbours of $y$ and $z$ in $\Gamma_{3}$, hence they sum to $t-2$. By (12a), $s_{13}^{1}=s_{23}^{2}=(t-2) / 2$. This is independent of $z$, thus $x$ is adjacent in $\Gamma_{1}$ to $t / 2$ vertices from each of the $r-1 t$-cliques of $\Gamma_{3}$ not containing $x$. Hence, $v_{1}=t(r-1) / 2=v_{2}$.

Now, $p_{12}^{1}=t-1$, and since this parameter is the sum of $s_{13}^{1}$ and $s_{23}^{1}$, we obtain $s_{23}^{1}=t / 2$. Next, $\beta_{12}^{1}=s_{13}^{1}-s_{23}^{1}=-1$. We determine $\beta_{11}^{2}$ using this and $k=$ $v_{1}+v_{3}, l=v_{2}$, obtaining

$$
\beta_{11}^{2}=-\frac{t(r-1)}{t-1}
$$

which, incidentally, implies that $t-1$ must divide $r-1$.

Finally, $\beta_{22}^{2}=p_{22}^{2}=s_{33}^{3}=t-2$. The weighted adjacency matrices are therefore determined.

$$
\mathbf{M}_{1}^{\omega}=\left(\begin{array}{ccc}
1 & \\
t(r-1) & \beta_{11}^{1} & -\frac{t(r-1)}{t-1} \\
& -1 &
\end{array}\right) \quad \mathbf{M}_{2}^{\omega}=\left(\begin{array}{rrr} 
& 1 \\
& -1 & \\
t-1 & & t-2
\end{array}\right)
$$

Claim $s_{11}^{2}=\frac{t}{2}(r-2)-s_{11}^{1}$. To see this, consider $(x, y) \in \Gamma_{1}$ and let $C_{x}, C_{y}$ be the cliques of $\Gamma_{3}$ containing $x$ and $y$ respectively. Count the neighbours of $x$ in $\Gamma_{1}$ that are not contained in $C_{y}$. There are $t / 2$ of them in each of the $r-2$ cliques. We may also 
count these vertices as $s_{11}^{1}+s_{12}^{1}$ which proves the claim, given $v_{1}=v_{2}$. The parameters of these imprimitive, non-metric rank 4 schemes are therefore determined by $t$ and $r$, with the exception of $s_{11}^{1}$. The intersection matrices and character-multiplicity table are given below, with $x_{1}$ and $x_{2}$ roots of

$$
x^{2}+\left[2 s_{11}^{2}-\frac{t}{2}(r-2)\right] x-\frac{t^{2}(r-1)}{4(t-1)}
$$

and multiplicities

$$
\begin{aligned}
& z_{i}=\frac{r t^{2}(r-1)(t-1)}{2 t^{2}(r-1)+4 u x_{i}(t-1)}, \quad u=2 s_{11}^{2}-\frac{t}{2}(r-2) . \\
& \mathbf{M}_{1}=\left(\begin{array}{cccc}
\frac{t(r-1)}{2} & s_{11}^{1} & s_{11}^{2} & \frac{t(r-1)(t-2)}{4(t-1)} \\
& s_{11}^{2} & s_{11}^{1} & \frac{t^{2}(r-1)}{4(t-1)} \\
& \frac{t-2}{2} & \frac{t}{2} &
\end{array}\right) \\
& \mathbf{M}_{2}=\left(\begin{array}{rrrr} 
& & 1 & \\
& s_{11}^{2} & s_{11}^{1} & \frac{t^{2}(r-1)}{4(t-1)} \\
\frac{t(r-1)}{2} & s_{11}^{1} & s_{11}^{2} & \frac{t(r-1)(t-2)}{4(t-1)} \\
& \frac{t}{2} & \frac{t-2}{2} &
\end{array}\right) \quad \mathbf{M}_{3}=\left(\begin{array}{rrrr} 
& & 1 \\
& \frac{t-2}{2} & \frac{t}{2} & \\
& \frac{t}{2} & \frac{t-2}{2} & \\
& & & t-2
\end{array}\right) \\
& \begin{array}{c|cccc|c} 
& \mathbf{I} & \mathbf{A}_{1} & \mathbf{A}_{2} & \mathbf{A}_{3} & m_{i} \\
\hline \zeta_{1} & 1 \frac{t}{2}(r-1) & \frac{t}{2}(r-1) & t-1 & 1
\end{array}
\end{aligned}
$$

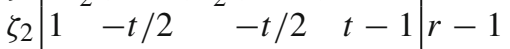

$$
\begin{aligned}
& \begin{array}{l|llll|l}
\zeta_{3} & 1 & x_{1} & -x_{1} & -1 & z_{1} \\
\zeta_{4} & 1 & x_{2} & -x_{2} & -1 & z_{2}
\end{array}
\end{aligned}
$$

We have observed that each vertex is adjacent in $\Gamma_{1}$ to exactly half, that is $t / 2$, of the vertices in each $t$-clique of $\Gamma_{3}$. Of course, it is adjacent to the remaining $t / 2$ vertices in $\Gamma_{2}$. Chang and Huang use separate parameters, $a$ and $b$, to represent the cardinalities of the intersections of $\Gamma_{1}(x)$ and $\Gamma_{2}(x)$ with a clique not containing $x$. Thus the rank 4 ISAs do not necessarily have $v_{1}=v_{2}$.

Theorem 2 A rank 4 ISA is the coherent closure of a regular weight on a strongly regular graph if and only if $a=b$.

Proof A rank 4 ISA has $s_{21}^{3} \neq 0$, hence $v_{1}=v_{2}$ by Lemma 4 if it is the CCL of a regular weight. Conversely, if $a=b$ in the matrices of [7], a simple calculation of the parameters for the weighted fusion $\left\{1^{-} 2^{+}\right\}\left\{3^{+}\right\}$shows that it is coherent. By Proposition 1 it is regular. 
Central to the work of Chang and Huang are the Higmanian SRGs that occur as $\Gamma_{1}$ or $\Gamma_{2}$ in a rank 4 ISA. The ISA is said to be of strongly regular type when either of these is strongly regular (and primitive).

Theorem 3 ([7, Theorem 3.1]) The vertices of a Higmanian SRG may be partitioned into cocliques meeting the Hoffman bound.

Remark The Hoffman cocliques are in fact the maximal cliques of $\Gamma_{3}$.

When an ISA is the closure of a regular weight, we may check using the intersection matrices in this section to see whether it is strongly regular type. If $\Gamma_{1}$ is strongly regular, then the fusion $\{1\}\{23\}$ must be coherent, and this requires

$$
s_{11}^{1}=\frac{t}{2}\left(\frac{t(r-1)}{2(t-1)}-1\right)
$$

Similarly, $\Gamma_{2}$ is strongly regular when

$$
s_{11}^{1}=\frac{t(r-1)(t-2)}{4(t-1)}
$$

and both conditions hold if and only if $r=t$.

Lemma 6 A Higmanian SRG $\Gamma$ arises from the coherent closure of a weighted SRG only if $\Gamma$ lies in the switching class of a regular two-graph.

Proof By Theorem 2 we know $a=b$ in the associated ISA. But a Higmanian SRG has parameters $(n, k ; r, s)=(n, t a ; t b / v,-a)$ or $(n, t b ; b,-t a / v)$, from [7, Sect. 2]. Substituting $a=b$ we find

$$
(n, k, \lambda, \mu)=\left(2(k-s), k, \frac{s(k+2 s+1)}{2 s+1}, \frac{k(s+1)}{2 s+1}\right) \text {, }
$$

and these SRG parameters satisfy the conditions of Proposition 2.

It is a simple task to check directly that the weighted fusion $\left\{1^{-} 2^{+} 3^{+}\right.$gives a regular two-graph if and only if $\Gamma_{1}$ is an SRG. However, the converse of Lemma 6 is false, as there exist Higmanian SRGs with $a \neq b$ that lie in the switching classes of regular two-graphs. The latin square parameters $L_{1,2}(5)$ yield two such schemes.

Some SRGs with the parameters of Lemma 6 are given in Table 1. Not all are Higmanian. The two-graph condition is not sufficient, as evidenced by SRGs in this list which are Higmanian but do not arise as closures. "Non-Higmanian" indicates that there is no rank 4 scheme containing the SRG; for the Higmanian entries we indicate by "closure" if the rank 4 scheme is the CCL of a regular weight. We make no claim about existence here. 
Table 1 SRGs in regular two-graphs

\begin{tabular}{|c|c|}
\hline$(n, k, \lambda, \mu)$ & Type \\
\hline$(10,3,0,1)$ & Non-Higmanian \\
\hline$(16,5,0,2)$ & Amorphic \\
\hline$(16,6,2,2)$ & Amorphic, closure $L_{1,2}(4)$ \\
\hline$(26,10,3,4)$ & Non-Higmanian \\
\hline$(28,12,6,4)$ & Higmanian, closure \\
\hline$(36,14,4,6)$ & Non-Higmanian \\
\hline$(36,15,6,6)$ & $\begin{array}{l}\text { Amorphic, closure } L_{1,3}(6) \text {; also in } \\
L_{2,2}(6) ; \text { neither exists }\end{array}$ \\
\hline$(50,21,8,9)$ & Non-Higmanian \\
\hline$(64,27,10,12)$ & $\begin{array}{l}\text { Amorphic, occurs in another } \\
\text { primitive scheme }\end{array}$ \\
\hline$(64,28,12,12)$ & $\begin{array}{l}\text { Amorphic, closure } L_{1,4}(8) \text {, occurs in } \\
\text { primitive } L_{2,3}(8)\end{array}$ \\
\hline$(76,30,8,14)$ & Non-Higmanian \\
\hline$(76,35,18,4)$ & Non-Higmanian \\
\hline$(82,36,15,16)$ & Non-Higmanian \\
\hline$(96,45,24,18)$ & $\begin{array}{l}\text { Higmanian, closure, occurs also in } \\
\text { primitive scheme }\end{array}$ \\
\hline$(100,44,18,20)$ & $\begin{array}{l}\text { Amorphic, occurs also in primitive } \\
\text { scheme }\end{array}$ \\
\hline$(100,45,20,20)$ & $\begin{array}{l}\text { Amorphic, closure } L_{1,5}(10) \text {, occurs } \\
\text { in } L_{2,4}(10), L_{3,3}(10)\end{array}$ \\
\hline
\end{tabular}

\subsubsection{Amorphic schemes}

Suppose now that both $\Gamma_{1}$ and $\Gamma_{2}$ are strongly regular (so we have an amorphic scheme, allowing that $\Gamma_{3}$ is not connected). By Theorem 4.1 of [30], the SRG parameters for all three graphs have Latin square type:

$$
\left(n^{2}, l_{i}(n-1), n-2+\left(l_{i}-1\right)\left(l_{i}-2\right), l_{i}\left(l_{i}-1\right)\right), i=1,2,3
$$

or negative Latin square type:

$$
\left(n^{2}, l_{i}(n+1),-n-2+\left(l_{i}+1\right)\left(l_{i}+2\right), l_{i}\left(l_{i}+1\right)\right), i=1,2,3 .
$$

In our case, negative type is ruled out because $v_{3}=t-1=n-1$. This implies also that $l_{3}=1$. From $\mathbf{M}_{1}$ we see that $\Gamma_{1}$ must have parameters $\{\lambda, \mu\}=\left\{s_{11}^{1}, s_{11}^{2}=n(n-2) / 4\right\}$ and $k=n(n-1) / 2$. Then $l_{1}=n / 2$ and we obtain

$$
\Gamma_{1}=\operatorname{SRG}\left(n^{2}, \frac{n(n-1)}{2}, \frac{n(n-2)}{4}, \frac{n(n-2)}{4}\right) .
$$

We see, similarly, that $\Gamma_{2}$ has the same parameters. 
Theorem 4 An amorphic rank 4 scheme is the coherent closure of a weighted SRG if and only if it has Latin square type $L_{1, m}(2 m)$.

Proof A Latin square scheme $L_{1, m}(2 m)$ as defined in [30] has two relations with SRG parameters

$$
\left(4 m^{2}, m(2 m-1), m(m-1), m(m-1)\right)
$$

and the third a disjoint union of complete graphs.

\begin{tabular}{c|cccc|c} 
& $\mathbf{I}$ & $\mathbf{A}_{1}$ & $\mathbf{A}_{2}$ & $\mathbf{A}_{3}$ & $m_{i}$ \\
\hline$\zeta_{1}$ & 1 & $m(2 m-1)$ & $m(2 m-1)$ & $2 m-1$ & 1 \\
$\zeta_{2}$ & 1 & $-m$ & $-m$ & $2 m-1$ & $2 m-1$ \\
$\zeta_{3}$ & 1 & $-m$ & $-m$ & -1 & $m(2 m-1)$ \\
$\zeta_{4}$ & 1 & $-m$ & $-m$ & -1 & $m(2 m-1)$
\end{tabular}

Substituting $n=2 m$ in the argument above shows that an amorphic CCL has this type. Conversely, given a Latin square type scheme with the stated parameters, the intersection matrices with an appropriate ordering are the $\mathbf{M}_{i}$ given in this section, with $t=r=n$ and $s_{11}^{1}=n(n-2) / 4$. From these it is clear that the scheme fuses to a regular weight on $\Gamma_{1}+\Gamma_{2}$ with

$$
\mathbf{M}_{1}^{\omega}=\left(\begin{array}{rrr}
1 & \\
n(n-1) & 0 & -n \\
-1 &
\end{array}\right) \quad \text { and } \quad \mathbf{M}_{2}^{\omega}=\left(\begin{array}{llr} 
& 1 \\
n-1 & & n-2
\end{array}\right)
$$

\subsection{Case $\mathbf{s}_{\mathbf{2 3}}^{\mathbf{1}}=\mathbf{0}$}

Suppose now that $s_{23}^{1}=0$. Immediately, $\beta_{12}^{1}=p_{12}^{1}=s_{13}^{1}=t-1, \beta_{11}^{2}=t(r-1)$. As before, $\beta_{22}^{2}=p_{22}^{2}=s_{33}^{3}=t-2$. Again let $t$ be the size of the $\Gamma_{3}$-cliques. Given a vertex $x$ and a clique $C$ not containing $x$, we see that $x$ has either no $\Gamma_{1}$ neighbours in $C$, or is adjacent to every vertex in $C$. The same is true for $\Gamma_{2}$, thus the quotient of this rank 4 scheme by $\Gamma_{0} \cup \Gamma_{3}$ is well defined, and yields an SRG with $(\tilde{n}, \tilde{k}, \tilde{\lambda}, \tilde{\mu})=\left(r, a, s_{11}^{1} / t, s_{11}^{2} / t\right)$. Here $\Gamma_{3}$ is a union of $r$ cliques of size $t$, and $a$ is determined by $v_{1}=a t$. We find $v_{2}=t(r-a-1)$. Observe, $v_{1} \neq v_{2}$, since $\beta_{12}^{1}=\beta_{11}^{2} \Longrightarrow t-1=t(r-1)$. This implies $t(r-2)=-1$, and the only solution is $t=r=1$.

The weighted adjacency matrices are

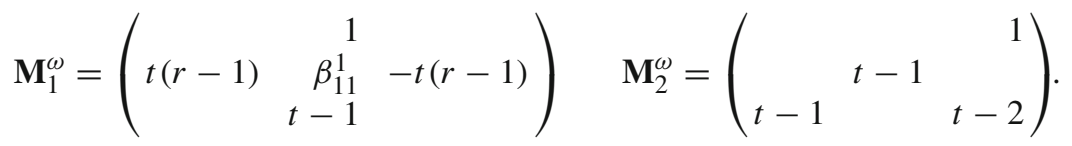


Example 1 The Petersen graph $\operatorname{SRG}(10,3,0,1)$ is obtained as a quotient from a scheme on 30 points with $t=3, a=3, v_{1}=9, v_{2}=18$, which also fuses to a weighted SRG, the complement of $\Gamma_{3}$.

Substituting $s_{12}^{1}=s_{11}^{2} v_{2} / v_{1}$ into (11a) we get

$$
s_{12}^{1}=s_{11}^{2} \frac{r-1-a}{a} .
$$

So

$$
\frac{2(r-1-a)}{a} s_{11}^{2}=s_{11}^{2}+s_{22}^{2}
$$

and from this we obtain

$$
s_{22}^{2}=\frac{2 r-2-3 a}{a} s_{11}^{2} .
$$

However, we may compute $s_{22}^{2}$ using the SRG parameters (i.e., this is the $\mu$ parameter, divided by $t$, for the complement of the quotient by $\Gamma_{3}$ ). We obtain

$$
\begin{gathered}
s_{22}^{2}=\frac{t(r-2 a-2)(2 r-3 a-2)}{2(r-2 a-1)}, \quad s_{12}^{1}=\frac{t(r-a-1)(r-2 a-2)}{2(r-2 a-1)}, \\
\quad \text { and } s_{11}^{2}=\frac{a t(r-2 a-2)}{2(r-2 a-1)} .
\end{gathered}
$$

Note that $r-2 a-1 \neq 0$ since $v_{1} \neq v_{2}$. Since $r-2 a-1$ is relatively prime to $r-2 a-2$, it divides both $t(r-a-1)$ and $a t$, and therefore divides $t(r-1)$.

In a similar way, we determine

$$
s_{11}^{1}=\frac{t(r-2 a)(3 a-r+1)}{2(r-2 a-1)} \quad \text { and } \quad s_{22}^{1}=\frac{t(r-2 a)(r-a-1)}{2(r-2 a-1)} .
$$

Hence, $\tilde{\Gamma}$ satisfies

$$
\tilde{\lambda}=\frac{(\tilde{n}-2 \tilde{k})(3 \tilde{k}-\tilde{n}+1)}{2(\tilde{n}-2 \tilde{k}-1)}, \quad \tilde{\mu}=\frac{\tilde{k}(\tilde{n}-2 \tilde{k}-2)}{2(\tilde{n}-2 \tilde{k}-1)} .
$$

Theorem 5 If $\mathcal{S}=\operatorname{CCL}\left(\mathcal{A}^{\omega}\right)$ is a rank 4 association scheme with notation as above and $s_{23}^{1}=0$, then $\mathcal{S}$ is $K_{t} \imath \tilde{\Gamma}$, with $\tilde{\Gamma}=\operatorname{SRG}(\tilde{n}, \tilde{k}, \tilde{\lambda}, \tilde{\mu})=$ as above, $\tilde{n}=r$, and $\tilde{k}=a$.

Example 2 The SRGs meeting these conditions include the Petersen graph (10,3, $0,1)$; the Clebsch graph $(16,5,0,2)$; the Lattice and Shrikhande graphs $(16,6,2,2)$; the Paulus graph $(26,10,3,4)$; the Chang and the $T(8)$ graphs $(28,12,6,4)$; and the complete bipartite graphs. See $[4,15,20,21]$ for more. 


\section{Main theorem}

We are now able to classify the rank 4 CCLs into the types depicted in Fig. 3, namely: (I) distance-regular Taylor graph schemes, (II) amorphic schemes, (III) Higmanian but not amorphic schemes, and (IV) non-Higmanian schemes, in which both $\mathbf{A}_{1}$ and $\mathbf{A}_{2}$ have four distinct eigenvalues.

Theorem 6 Let $\mathcal{B}$ be the coherent closure of a strongly regular graph $\Gamma$ with a regular weight $\omega$, and notation as above. If $\mathcal{B}$ has rank 4 then it is symmetric and imprimitive with one relation, say $\Gamma_{3}$, a disjoint union of cliques of size $t>1$. Then

1. $\mathcal{B}$ is metric if and only if $t=2$ and $s_{23}^{1} \neq 0$, and in that case $\Gamma_{1}$ is an antipodal distance-regular graph with distance array $\left\{b_{0}, b_{1}, 1 ; 1, b_{1}, b_{0}\right\}$.

2. $\mathcal{B}$ is a wreath product if and only if $s_{23}^{1}=0$, and in that case $\mathcal{B}$ is $K_{t} 2 \tilde{\Gamma}, \tilde{\Gamma}$ is $\operatorname{SRG}(\tilde{n}, \tilde{k}, \tilde{\lambda}, \tilde{\mu})$ as in Theorem 5.

3. $\mathcal{B}$ is Higmanian if and only if $\Gamma_{1}$ or $\Gamma_{2}$ is strongly regular with Hoffman partition given by the connected components of $\Gamma_{3}$ and lies in the switching class of a regular two-graph.

4. $\mathcal{B}$ is amorphic if and only if it has latin square type $L_{1, m}(2 m)$.

5. If none of the above, then $\Gamma_{1}$ and $\Gamma_{2}$ each have 4 distinct eigenvalues and are cospectral unless all eigenvalues are integral.

Proof The first statement follows from Lemma 4. For (1), Theorem 1 supplies one direction. On the other hand, suppose $t=2$ in an ISA scheme, and observe that $\mathbf{M}_{1}$ is tri-diagonal, with $s_{11}^{2} \neq 0$ and $s_{23}^{1} \neq 0$. This implies that $\mathcal{B}$ is metric, and the parameters $b_{i}$ and $c_{i}$ are given by $s_{i+1,1}^{i}$ and $s_{i-1,1}^{i}$ respectively, resulting in the stated distance array.

Part (2) is Theorem 5 combined with the observation that $s_{23}^{1}$ must be 0 in a wreath product given that $\mathbf{B}_{1}=\mathbf{A}_{1} \otimes \mathbf{J}, \mathbf{B}_{2}=\mathbf{A}_{2} \otimes \mathbf{J}, \mathbf{B}_{3}=\mathbf{I} \otimes(\mathbf{J}-\mathbf{I})$ with vertices and indices ordered suitably.

Part (3) follows from [7, Thm. 3.1] and Lemma 6; part (4) from Theorem 4.

To prove (5), we suppose $\mathcal{B}$ is neither Higmanian nor a wreath product. Then both $\Gamma_{1}$ and $\Gamma_{2}$ have four distinct eigenvalues and $s_{23}^{1} \neq 0$ so we are in the case of Sect. 8.2. The eigenvalues of $\Gamma_{1}$ and $\Gamma_{2}$ are $\pm x_{1}, \pm x_{2}$, where $x_{1}$ and $x_{2}$ are the roots of (15). Since $z_{i} \in \mathbb{Z}$, we see by (16) that either $x_{1}$ and $x_{2}$ are rational, in which case they are integers as are the remaining eigenvalues (shown in the character-multiplicity table), or $2 s_{11}^{2}-\frac{t}{2}(r-2)=0$. In the latter case, the coefficient of $x$ in (15) is 0 , so $x_{1}=-x_{2}$ and we see that $\Gamma_{1}$ and $\Gamma_{2}$ are cospectral.

\subsection{Parameters}

Using tables of rank 4 association schemes from [30] and personal communication with E. R. van Dam, we list the feasible parameters for rank 4 CCLs of weighted SRGs on up to 100 vertices for the Taylor graphs and 200 for the others, along with the number of schemes known. A '?' in the table indicates that the number of schemes is unknown. We refer to [30] and the citations therein for additional information and for clarity on the notations used in the final column. 
Table 2 Parameters of rank 4 closures

\begin{tabular}{|c|c|c|c|c|c|c|c|}
\hline $\mathrm{n}$ & $\mathrm{t}$ & $\mathrm{r}$ & $\mathrm{s}_{11}^{1}$ & Type & Cospectral & $\#$ & Notes \\
\hline 6 & 2 & 3 & 0 & I & & 1 & $C_{6} \simeq R(3,2)$ \\
\hline 8 & 2 & 4 & 0 & I & & 1 & Cube $\simeq R(4,2)$ \\
\hline 20 & 2 & 10 & 4 & I & $\mathrm{y}$ & 1 & $J(6,3)$ \\
\hline 32 & 2 & 16 & 6 & I & & 1 & $2(G Q(2,2)+1)$ \\
\hline 52 & 2 & 26 & 12 & I & $\mathrm{y}$ & 4 & \\
\hline 56 & 2 & 28 & 10 & I & & 1 & 2(Schlafli+1) \\
\hline 72 & 2 & 36 & 16 & I & & $\geq 227$ & \\
\hline 90 & 2 & 45 & 18 & I & & 0 & \\
\hline 100 & 2 & 50 & 24 & I & $\mathrm{y}$ & $\geq 18$ & $2(P(49)+1)$ \\
\hline 4 & 2 & 2 & 0 & II & $\mathrm{y}$ & 1 & $L_{1,1}(2) \simeq R(2,2)$ \\
\hline 16 & 4 & 4 & 2 & II & $\mathrm{y}$ & 4 & $L_{1,2}(4)$ \\
\hline 36 & 6 & 6 & 6 & II & $\mathrm{y}$ & 0 & $L_{1,3}(6)$ \\
\hline 64 & 8 & 8 & 12 & II & $\mathrm{y}$ & $\geq 1$ & $L_{1,4}(8)$ \\
\hline $4 m^{2}$ & $2 m$ & $2 m$ & $m^{2}-m$ & II & $\mathrm{y}$ & $?$ & $L_{1, m}(2 m), m \geq 5$ \\
\hline 28 & 4 & 7 & 4 & III & & 56 & From $T(8)$, Chang \\
\hline 96 & 6 & 16 & 18 & III & & $?$ & \\
\hline 120 & 8 & 15 & 24 & III & & $?$ & \\
\hline 126 & 6 & 21 & 24 & III & & $?$ & \\
\hline 190 & 10 & 19 & 40 & III & & $?$ & \\
\hline 40 & 4 & 10 & 8 & IV & $\mathrm{y}$ & 0 & \\
\hline 64 & 4 & 16 & 14 & IV & $\mathrm{y}$ & $?$ & \\
\hline 88 & 4 & 22 & 20 & IV & $\mathrm{y}$ & 0 & \\
\hline 100 & 4 & 25 & 21 & IV & & $?$ & \\
\hline 112 & 4 & 28 & 26 & IV & $\mathrm{y}$ & $?$ & \\
\hline 136 & 4 & 34 & 32 & IV & $\mathrm{y}$ & $?$ & \\
\hline 156 & 6 & 26 & 36 & IV & $\mathrm{y}$ & $?$ & \\
\hline 160 & 4 & 40 & 38 & IV & $\mathrm{y}$ & $?$ & \\
\hline 176 & 8 & 22 & 39 & IV & & $?$ & \\
\hline 176 & 8 & 22 & 40 & IV & $\mathrm{y}$ & $?$ & \\
\hline 184 & 4 & 46 & 44 & IV & $\mathrm{y}$ & $?$ & \\
\hline 186 & 6 & 31 & 42 & IV & & $?$ & \\
\hline
\end{tabular}

Acknowledgments This work began in earnest at the Banff International Research Station during the Workshop on Algebraic Graph Theory in April 2011, and was completed while visiting Tilburg University during the 2012-2013 academic year. Many thanks to the organizers and sponsors of the former, for the opportunity to attend, and to the Department of Econometrics and Operations Research, especially Edwin van Dam and Willem Haemers at the latter, for their kind and generous hospitality. Thanks are due to Edwin van Dam for assistance on three-class schemes. Eric Webster wrote C++ and GAP code to test schemes for coherent weighted fusions. Michael Freeze wrote sage code to test SRG parameters for feasible weights and CCLs of small rank. 


\section{References}

1. Babel, L., Chuvaeva, I., Klin, M., Pasechnik, D.: Algebraic combinatorics in mathematical chemistry. Methods and algorithms. II. Program implementation of the Weisfeiler-Lehman algorithm. arXiv:1002.1921 [math.CO]

2. Bannai, E., Ito, T.: Algebraic Combinatorics. I: Association Schemes. The Benjamin/Cummings Publishing Co., Inc., Menlo Park, CA (1984)

3. Brouwer, A.: BCN tables. http://www.win.tue.nl/ aeb/drg/drgtables.html. Accessed 2012

4. Brouwer, A.: Parameters of strongly regular graphs. http://www.win.tue.nl/ aeb/graphs/srg/srgtab. html. Accessed 2013

5. Brouwer, A.E., Cohen, A.M., Neumaier, A.: Distance-Regular Graphs. Springer, Berlin (1989)

6. Cameron, P.J., van Lint, J.H.: Designs, Graphs, Codes and Their Links, London Mathematical Society Student Texts, vol. 22. Cambridge University Press, Cambridge and New York (1991)

7. Chang, Y., Huang, T.: Imprimitive association schemes of low ranks and Higmanian graphs. Ann. Comb. 4, 317-326 (2000)

8. Conway, J.H., Curtis, R.T., Norton, S.P., Parker, R.A., Wilson, R.: Atlas of Finite Groups. Clarendon Press, Oxford (1985)

9. Godsil, C.D.: Algebraic Combinatorics. Chapman and Hall Mathematics Series. Chapman \& Hall, New York (1993)

10. Hanaki, A., Miyamoto, I.: Classification of association schemes of small order. http://kissme.shinshu-u. ac.jp/as/. Accessed 2010-2011

11. Higman, D.G.: Coherent configurations I: ordinary representation theory. Geom. Dedicata 4, 1-32 (1975)

12. Higman, D.G.: Coherent configurations II: weights. Geom. Dedicata 5, 413-424 (1976)

13. Higman, D.G.: Coherent algebras. Linear Algebra Appl. 93, 209-239 (1987)

14. Higman, D.G.: Weights and $t$-graphs. Algebra, groups and geometry. Bull. Soc. Math. Belg. Sér. A 42(3), 501-521 (1990)

15. Jørgensen, L.K., Klin, M.H.: Switching of edges in strongly regular graphs. I.: a family of partial difference sets on 100 vertices. Electron. J. Comb. 10, R17 (2003)

16. Klin, M., Munemasa, A., Muzychuk, M., Zieschang, P.H.: Directed strongly regular graphs obtained from coherent algebras. Linear Algebra Appl. 377, 83-109 (2004)

17. Klin, M., Muzychuk, M., Pech, C., Woldar, A., Zieschang, P.H.: Association schemes on 28 points as mergings of a half-homogeneous coherent configuration. Eur. J. Comb. 28(7), 19942025 (2007)

18. Maple 16.00: Maplesoft, a division of Waterloo Maple Inc., Waterloo, ON (2013)

19. Martin, W.J., Tanaka, H.: Commutative association schemes. Eur. J. Comb. 30, 1497-1524 (2009)

20. McKay, B.D., Spence, E.: Classification of regular two-graphs on 36 and 38 vertices. Australas. J. Comb. 24, 293-300 (2001)

21. Mesner, D.M.: Negative Latin Square Designs, NC Mimeo Series, vol. 410. Institute of Statistics, UNC. Chapel Hill, NC (1964)

22. Sankey, A.D.: Regular weights on strongly regular graphs. Ph.D. thesis, University of Michigan, Ann Arbor, MI (1992)

23. Sankey, A.D.: Regular weights of full rank on strongly regular graphs. Isr. J. Math. 95, 1-23 (1996)

24. Seidel, J.J.: A survey of two-graphs. In: Colloquio Internazionale sulle Teorie Combinatorie (Rome, 1973), Tomo I, pp. 481-511. Atti dei Convegni Lincei, No. 17. Accad. Naz. Lincei, Rome (1976)

25. Seidel, J.J.: More about two-graphs. In: Fourth Czechoslovakian Symposium on Combinatorics, Graphs and Complexity (Prachatice, 1990), Annals of Discrete Mathematics, vol. 51, pp. 297-308. NorthHolland, Amsterdam (1992)

26. Seidel, J.J., Taylor, D.E.: Two-graphs, a second survey. In: Algebraic Methods in Graph Theory, vols. I, II (Szeged, 1978), Colloquia Mathematica Societatis János Bolyai, vol. 25, pp. 689-711. NorthHolland, Amsterdam (1981)

27. Stein, W.: Sage: Open Source Mathematical Software (Version 2.10.2). http://www.sagemath.org (2008)

28. Taylor, D.E.: Regular 2-graphs. Proc. Lond. Math. Soc. Ser. 3. 35(2), 257-274 (1977)

29. The GAP Group: Gap-Groups, Algorithms, and Programming (Version 4.6.4). http://www. gap-system.org (2013)

30. van Dam, E.R.: Three-class association schemes. J. Algebr. Comb. 10, 69-107 (1999) 
31. van Dam, E.R., Koolen, J.H., Tanaka, H.: Distance-regular graphs. https://sites.google.com/site/ edwinrvandam/. Accessed Oct 2012

32. Weisfeiler, B., Lehman, A.: Reduction of a graph to a canonical form and an algebra which appears in the process. NTI 9, 12-16 (1968) 